\title{
The link between molecular cloud structure and turbulence
}

\author{
N. Schneider ${ }^{1}$, S. Bontemps ${ }^{2}$, R. Simon ${ }^{3}$, V. Ossenkopf ${ }^{3}$, C. Federrath ${ }^{4,5,6}$, R. S. Klessen ${ }^{4,7}$, F. Motte ${ }^{1}$, Ph. André ${ }^{1}$, \\ J. Stutzki ${ }^{3}$, and C. Brunt ${ }^{8}$
}

1 IRFU/SAp CEA/DSM, Laboratoire AIM CNRS - Université Paris Diderot, 91191 Gif-sur-Yvette, France e-mail: nschneid@cea.fr

2 OASU/LAB-UMR5804, CNRS, Université Bordeaux 1, 33270 Floirac, France

3 I. Physikalisches Institut, Universität zu Köln, Zülpicher Straße 77, 50937 Köln, Germany

${ }^{4}$ Zentrum für Astronomie der Universität Heidelberg, Inst. für Theor. Astrophysik, Albert-Ueberle Str. 2, 69120 Heidelberg, Germany

5 Max-Planck-Institut für Astronomie, Königstuhl 17, 69117 Heidelberg, Germany

${ }^{6}$ École Normale Supérieure de Lyon, CRAL, 69364 Lyon Cedex 07, France

7 Kavli Institute for Particle Astrophysics and Cosmology, Stanford University, Menlo Park, CA 94025, USA

8 School of Physics, University of Exeter, Exeter EX4QL, UK

Received 16 December 2009 / Accepted 18 December 2010

\section{ABSTRACT}

\begin{abstract}
Aims. We aim to better understand how the spatial structure of molecular clouds is governed by turbulence. For that, we study the large-scale spatial distribution of low-density molecular gas and search for characteristic length scales.

Methods. We employ a 35 square degrees ${ }^{13} \mathrm{CO} 1 \rightarrow 0$ molecular line survey of Cygnus $\mathrm{X}$ and visual extinction $\left(\mathrm{A}_{\mathrm{V}}\right)$ maps of 17 Galactic clouds to analyze the spatial structure with the $\Delta$-variance method. This sample contains a large variety of different molecular cloud types with different star-forming activity.

Results. The $\Delta$-variance spectra obtained from the $\mathrm{A}_{\mathrm{V}}$ maps show differences between low-mass star-forming (SF) clouds and massive giant molecular clouds (GMC) in terms of shape of the spectrum and its power-law exponent $\beta$. Low-mass SF clouds have a double-peak structure with characteristic size scales around $1 \mathrm{pc}$ (though with a large scatter around this value) and $4 \mathrm{pc}$. The GMCs show no characteristic scale in the $A_{V}$-maps, which can partly be ascribed to a distance effect owing to a larger line-of-sight (LOS) confusion. The $\Delta$-variance for Cygnus, determined from the ${ }^{13} \mathrm{CO}$ survey, shows characteristic scales at $4 \mathrm{pc}$ and $40 \mathrm{pc}$, either reflecting the filament structure and large-scale turbulence forcing or - for the $4 \mathrm{pc}$ scale - the scale below which the ${ }^{13} \mathrm{CO} 1 \rightarrow 0$ line becomes optically thick. Though there are different processes that can introduce characteristic scales, such as geometry, decaying turbulence, the transition scale from supersonic to subsonic turbulence (the sonic scale), line-of-sight effects and energy injection caused by expanding supernova shells, outflows, HII-regions, and although the relative contribution of these effects strongly varies from cloud to cloud, it is remarkable that the resulting turbulent structure of molecular clouds shows similar characteristics.
\end{abstract}

Key words. methods: analytical - dust, extinction - ISM: molecules - ISM: structure

\section{Introduction}

\section{Turbulence}

Over the last years, much progress has been made to theoretically explain the observational claim that turbulence governs the different phases of the interstellar medium, (ISM; MacLow \& Klessen 2004; Elmegreen \& Scalo 2004; Scalo \& Elmegreen 2004). Molecular line surveys of molecular clouds as a whole led to the detection of the linewidth-size $\sigma \propto L^{\epsilon 1}$ and mass-size $M \propto L^{\gamma}$ relations (Larson 1981) with $\epsilon \sim 0.2 \ldots 0.5$ and $\gamma \sim 2$ (e.g. Goldsmith et al. 1987; Heyer \& Brunt 2004; Heyer et al. 2009; Lombardi et al. 2010), and a mass distribution for molecular clumps of the form of $\mathrm{d} N / \mathrm{d} M \sim M^{-\alpha}$ with $\alpha \sim 1.5-1.9$ (e.g. Stutzki \& Güsten 1990; Kramer et al. 1998). The spatial structure of the emission has been characterized in terms of power spectra (Scalo 1987; Stutzki et al. 1998).

The scaling relations and power laws can be explained in the context of turbulence, where energy is injected at very large scales and cascades down to the smallest scales, creating eddies

\footnotetext{
${ }^{1}$ In the following, we use $\alpha$ for the clump-mass spectral index, $\beta$ for the slope of the $\Delta$-variance, $\gamma$ for the power-law exponent of the masssize relation, and $\epsilon$ for the power-law exponent of the linewidth-size relation.
}

and stirring up the cloud. In particular the linewidth-size relation of giant molecular clouds can only be reproduced if the ISM turbulence includes sources of driving that act on large scales, but small-scale driving by outflows can be important in localized (sub-parsec) regions of larger clouds. The driving sources for turbulence on the largest scales can be diverse, ranging from the accretion of gas of extragalactic origin (Klessen \& Hennebelle 2010) to the occurance of convergent flows of atomic gas triggered by spiral density waves (Walder \& Folini 1998; VázquezSemadeni et al. 2007; Heitsch et al. 2008; Hennebelle et al. 2008; Banerjee et al. 2009), supernova explosions (MacLow \& Klessen 2004; Dib et al. 2009), or expanding HII regions (Matzner 2002; Krumholz et al. 2006; Peters et al. 2008; Krumholz \& Matzner 2009; Gritschneder et al. 2009). Here it is the very process of cloud formation that drives the internal turbulence. Observations by Brunt et al. (2009) indicate that the size-velocity dispersion of giant molecular clouds can only be reproduced if the ISM turbulence is driven on large scales. Some models have investigated molecular cloud turbulence that is driven on small scales by internal sources such as stellar winds and outflows (Li \& Nakamura 2006; Nakamura \& Li 2008; Wang et al. 2010), but it is unlikely that these have a significant effect on the largest scales within clouds (Mac Low \& Klessen 2004; Banerjee et al. 2007; 
Brunt et al. 2009). All the above proposed driving sources will excite a considerable amount of compressive modes (Federrath et al. 2008), which are essential to trigger star formation.

In its simplest form, the Kolmogorov-turbulence (Kolmogorov 1941) describes an incompressible, non-magnetic fluid in which the statistical properties of the flow are independent of the scale within the inertial range. This leads to an index of $\epsilon=0.33$ and to a self-similar, i.e. fractal, spatial structure. Other turbulence fluid models (Vazquez-Semadeni et al. 1997; Ballesteros-Paredes \& MacLow 2002; Federrath et al. 2010) that describe a supersonic, isothermal turbulent flow obtain $\epsilon=0.5$ and $\gamma=2$. Hennebelle \& Audit (2007) and Hennebelle et al. (2007) take a different approach and describe turbulence by the individual motion of cloudlets. Their hydrodynamic, non-magnetic, non-self-gravitating simulations of a turbulent 2D-phase of atomic HI gas led to a mass-size relation of $M \sim L^{1.7}$ and a clump-mass spectral index $\alpha \approx 1.7$, a value similar to that found for CO clumps.

These few examples already show the effort that is made to take into account the complexity of the ISM and to compare observations with theory. However, each model represents a particular physical situation with a different focus on the various physical effects that play a role (driving source of turbulence, magnetic fields, self-gravity, thermal balance, etc.). In particular the importance of self-gravity was pointed out by, e.g. Klessen (2000), who discussed its effect on various statistical characteristics, and Vázquez-Semadeni et al. (2008), who emphasized that a part of the observed velocity dispersion in molecular clouds is caused by clump-scale inward motions as a consequence of gravitational collapse and not turbulence. Recently, Ballesteros-Paredes et al. (2011) have suggested that the size-linewidth-surface-density relation observed by Heyer et al. (2009) can be quantitatively explained if the internal turbulent motions are generated by hierarchical contraction. Their model successfully explains the apparent virialization of the clouds and potentially avoids the need for carefully balanced "replenishment" of the turbulence by "internal" driving sources. Early explanations of linewidths in molecular clouds (Goldreich \& Kwan 1974) as arising from gravitational collapse were opposed (Zuckerman \& Palmer 1974) on the basis of the untenably high star-formation rates that would ensue. Ballesteros-Paredes et al. (2011) propose that this problem may be avoided if the higher density regions collapse first (by virtue of their shorter free-fall times) and induce star-formation feedback, which interrupts the global collapse and quickly destroys the cloud. Recent hydrodynamic models (Krumholz 2006; Krumholz et al. 2007; Offner et al. 2009; Bate 2009) stress the importance of radiative feedback processes from stars that can drastically reduce the total number of stars formed, although it has been found that fragmentation is never completely suppressed (Peters et al. 2010a,b,c.) Similar considerations hold for the influence of magnetic fields in massive star-forming regions (Hennebelle et al. 2011; Peters et al. 2011).

These "deviations" from the idealized scenario of a purely turbulent medium may cause changes of the scaling laws. This could explain, for example, why many molecular line studies failed to detect a linewidth-size relation in molecular clumps (e.g. Loren 1989; Simon et al. 2001; Schneider \& Brooks 2004). In any case, it is difficult to transform model results into "observables" such as the 3D- or projected (on the plane of the sky) emission and velocity structure of an observed molecular line. Ballesteros-Paredes \& Mac Low (2002) argued that the apparent mass-size relation depends on the observations and/or the analysis process: any cutoff in column density automatically implies $M \propto L^{2}$ because of the limited dynamic range and/or the minimum intensity level defined by clump finding algorithms. Even more problematic is the velocity superposition of many clumps in the line-of-sight when a low-density tracer like ${ }^{12} \mathrm{CO}$ is used. High-density tracers like $\mathrm{CS}$ or $\mathrm{N}_{2} \mathrm{H}^{+}$provide a better clump seperation, but imply a bias in analyzing the full cloud structure (only the densest structures are identified). A survey using the ${ }^{13} \mathrm{CO}$ line may be the best compromise.

\section{Molecular cloud structure}

The observed spatial structure of molecular clouds depends on the tracer used to image the clouds. Low-J rotational transitions of ${ }^{12} \mathrm{CO}$ or visual extinction maps are sensitive to low densities and show a very complex structure that - with a change to higher angular resolution - breaks up into substructures, which appear similar on almost all scales (e.g., Falgarone et al. 1991; Stutzki 2001). The concept of "self-similarity" as a geometrical property was introduced (Elmegreen \& Falgarone 1996) and this gas component is well described as a fractal-Brownian-motion (fBM) structure (Stutzki et al. 1998). In contrast, gas at much higher column density, which is best traced by dust (sub)mmcontinuum emission or molecular line high density tracers like CS, shows no fractal structure (Falgarone et al. 2004). In addition, self-similarity may break down on the smallest scales $(<0.1 \mathrm{pc})$ which are relevant for star formation (e.g. Goodman et al. 1998; Dib et al. 2008; Federrath et al. 2010).

\section{Describing the molecular cloud structure}

Statistical tools have been developed to analyze the observed spatial and kinematic cloud structure, which turns out to be extremely complex. Amongst the most commonly used tools to describe structure are probability distribution functions of line intensities (Padoan et al. 1997), the $\Delta$-variance analysis of intensities and velocities (Stutzki et al. 1998; Ossenkopf 2002), the principal component analysis (Heyer \& Schloerb 1997; Brunt \& Heyer 2002a,b), and structure functions (Federrath et al. 2010). In the current study, we use the $\Delta$-variance method, applied to a large ${ }^{13} \mathrm{CO} 1 \rightarrow 0$ survey obtained with the $\mathrm{FCRAO}^{2}$ $14 \mathrm{~m}$ telescope of the Cygnus $\mathrm{X}$ region and on a data set of 17 near-IR extinction maps of molecular clouds derived from 2MASS. This analysis will help to further develop and refine models and cloud structure characterization techniques. We are particularly interested to see whether there is a dependence of cloud characteristics (size scales, value of the powerlaw slope $\beta$, form of the $\Delta$-variance spectrum) and cloud type (low- and high-mass star-forming molecular clouds, diffuse gas).

\section{Cygnus X}

The Cygnus $X$ region is the richest and most massive complex of high-mass star formation at a distance lower than $3 \mathrm{kpc}$. Schneider et al. $(2006,2007)$ showed that the Cygnus X region constitutes a large scale network of GMCs at a common distance of $\sim 1.7 \mathrm{kpc}$. Signposts of recent and ongoing (high-mass) star formation ( $\mathrm{H}$ in regions, outflow activity, IR-sources such as S106 IR, DR21, W75N, and GL2591) are ubiquitous. See Schneider et al. (2006) or Reipurth \& Schneider (2008) for a review on Cygnus $\mathrm{X}$. The region also contains several OB clusters (Cyg OB1-4, 6, and 8) including the richest known OB cluster of the Galaxy, Cyg OB2 (Knödlseder 2000, Uyaniker et al. 2001). Their energy injection from stellar winds and radiation could be a driving source for turbulence.

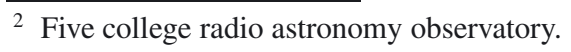




\section{Observations}

\subsection{FCRAO ${ }^{13} \mathrm{CO} 1 \rightarrow 0$ survey}

The data presented here were obtained with the FCRAO partly in remote operation from late 2003 to early 2006. The summer period was excluded for observations because of weather constraints. The molecular lines of ${ }^{13} \mathrm{CO} 1 \rightarrow 0$ at $110.201 \mathrm{GHz}$ and $\mathrm{C}^{18} \mathrm{O} 1 \rightarrow 0$ at $109.782 \mathrm{GHz}$ were observed simultaneously. The beamwidth of the FCRAO at $110 \mathrm{GHz}$ is $46^{\prime \prime}$.

A total of 35 square degrees in Cygnus $\mathrm{X}$ was covered in the ${ }^{13} \mathrm{CO}$ and $\mathrm{C}^{18} \mathrm{O} 1 \rightarrow 0$ lines at $46^{\prime \prime}$ angular resolution (Simon et al., in prep.). This survey is significantly larger and at higher angular resolution than the ${ }^{13} \mathrm{CO} 2 \rightarrow 1$ map obtained with $\mathrm{KOSMA}^{3}$, presented by Schneider et al. (2006). It has a spectral dynamic range (maximum observed linewidth over velocity resolution) of $\sim 70$ and a spatial dynamic range (map size over angular resolution) of $\sim 230$. This is much larger than what is usually obtained for molecular cloud maps (Kramer et al. 1998). The highest column density regions traced in the FCRAO ${ }^{13} \mathrm{CO}$ $1 \rightarrow 0$ survey were then observed in the $\mathrm{CS} 2 \rightarrow 1$ and $\mathrm{N}_{2} \mathrm{H}^{+} 1 \rightarrow 0$ lines ( $\sim 15$ square degrees) with the FCRAO.

Pointing and calibration were regularly checked at the start of the Cygnus observing time interval and after transit of Cygnus (no observations were performed at elevations higher than $75^{\circ}$ ). Pointing sources were $\mathrm{SiO}$ masers of evolved stars, i.e., $\chi$-Cyg, R-Leo and T-Cep, depending on observing time. The calibration was regularly checked on the position of peak emission in DR21 and found to be consistent within $10 \%$.

We used the single sideband focal plane array receiver SEQUOIA (Second Quabbin Optical Imaging Array; Erickson et al. 1999) designed for the $85-115.6 \mathrm{GHz}$ range, yielding a mean receiver noise temperature of $60 \mathrm{~K}$. SEQUOIA is a $4 \times 4$ dual-polarization array with a separation between elements of $88^{\prime \prime}$ on the sky. The receiver was used in combination with a dual channel correlator (DCC), configured to a bandwidth of 25 $\mathrm{MHz}$, using 1024 channels, corresponding to a velocity sampling of $0.066 \mathrm{~km} \mathrm{~s}^{-1}$.

We used the on-the-fly mapping mode (OTF) in which each map consisted of a block of $20^{\prime} \times 10^{\prime}$ (30 min integration time including overheads). The data quality is enhanced because pixelto-pixel variations are averaged in the final regridded data set. Since at the map edges the coverage is less dense, and thus leads to an increase in noise, adjacent maps were overlapping to cover the region at uniform density.

In total, 800000 spectra were produced on a $22^{\prime \prime} 5$ sampled grid covering an area of $35 \mathrm{deg}^{2}$. The data have a mean $1 \sigma_{\text {rms }}$ rms noise level of $\sim 0.2 \mathrm{~K}$ per $0.06 \mathrm{~km} \mathrm{~s}^{-1}$ channel on a $T_{\mathrm{A}}^{*}$ antenna temperature scale, i.e., not corrected for the main beam efficiency of $\sim 0.48$. More details on the observing procedure, regridding and the rms-map of ${ }^{13} \mathrm{CO}$ are found in Appendix A.

\subsection{Near-infrared extinction maps}

The basic idea of deriving extinction maps from near-infrared (near-IR) data is that the average reddening of background stars, as observed in the near-IR bands (usually $J, H$, and $K$ ) is used to estimate the total column density of dust along the line-of-sight and thus the total $A_{\mathrm{V}}$ in that direction (e.g. Lada et al. 1994).

We developed a new technique (AvMAP) to produce in an efficient way extinction maps from the $2 \mathrm{MASS}^{4}$ catalog following

\footnotetext{
3 Cologne observatory for Submm-Astronomy.

4 The Two Micron All Sky Survey (2MASS) is a joint project of the University of Massachusetts and the Infrared Processing and
}

the schemes described in Lombardi et al. (2001) and Cambresy et al. (2002), but with the improvements that (i) it is a fast algorithm that can easily handle large fields with millions of sources; (ii) it includes a calibration of the extinction that does not require an off-field; and (iii) it is a method to estimate the density of foreground stars that follows the spatial distribution of stars in the Galaxy by using the predictions of the stellar population model of Besançon (Robin et al. 2003; see http://model . obs-besancon. fr/) to filter out foreground stars. Thus, $A_{\mathrm{V}^{-}}$ maps can be used up to a distance of $3 \mathrm{kpc}$ at an angular resolution of typically $2^{\prime}$. A detailed description of the method is given in Appendix B.

\section{The $\Delta$-variance as a method of structure analysis}

\subsection{Definition of $\Delta$-variance}

The $\Delta$-variance is a method to analyze the structure and correlation in multi-dimensional scalar functions. It was first introduced by Stutzki et al. (1998), who expanded the 1-D Allan variance concept (Allan 1966) to two and more dimensions in order to characterize the observed 2D structure in molecular cloud images.

The $\Delta$-variance ${ }^{5}$ measures the amount of structure on a given scale $L$ in a map $s$ (2D scalar function) by filtering this map with a spherically symmetric wavelet $\bigcirc_{L}$ :

$\sigma_{\Delta}^{2}(L)=\left\langle\left(s \otimes \bigcirc_{L}\right)^{2}\right\rangle_{x, y}$.

The $\Delta$-variance thus probes the variation of the intensity $s$ over a length $L$ (called lag), i.e., the amount of structural variation on that scale. The filter function can vary between a smooth or a step-shaped filter function ("Mexican hat" or "French hat"). Ossenkopf et al. (2008a) systematically analyzed (using simulations) the influence of the shape of the filter function and arrived at the conclusion that the Mexican hat filter, with an annulus-tocore-diameter ratio of about 1.5 , provided the best results for a clear detection of pronounced scales.

The observed structure can be well mimicked by a fractional Brownian motion ( $\mathrm{fBm}$ ) structure (which has a power-law spectrum) in the context of fractal images. For any $2 D$ image with a power spectrum $P(k) \propto|k|^{-\beta}$, in which $k$ is the spatial frequency, the $2 \mathrm{D} \Delta$-variance varies as $\sigma_{\Delta}^{2} \propto L^{\beta-2}$ for $0<\beta<6$ (see Stutzki et al. 1998, for more details). The range of $L$ is given by the upper and lower spatial frequency cutoffs $k_{\mathrm{u}} \approx(\Delta x)^{-1}$ and $k_{1} \approx(n \Delta x)^{-1}\left(\Delta x\right.$ is the sampling grid of a map with $n^{2}$ pixels $)$ : $\left(2 \pi k_{\mathrm{u}}\right)^{-1}<L<\left(2 \pi k_{1}\right)^{-1}$.

The advantage of the $\Delta$-variance compared to the powerspectrum is that Fourier-based methods, like the determination of the power-spectrum, can create artifical structures by edge and gridding effects. This is not the case for the $\Delta$-variance, the size of the image only limits the largest lag probed and the shortest lag will be close to the angular resolution. In addition, noise contributions in the map can be separated from the intrinsic structure of the cloud because purely white noise has $\beta=0$ (flicker noise would lead to $\beta=1$ ). Weighting the image with the inverse noise function $(1 / \mathrm{rms})$ enables us to distinguish the variable noise from real small scale structure (see Bensch et al. 2001, for more details). At the smallest scales, the

Analysis Center/California Institute of Technology, funded by the National Aeronautics and Space Administration and the National Science Foundation.

5 All $\Delta$-variance calculations were performed with the IDL-based routine deltavar provided by V. Ossenkopf and are available at wWw. astro.uni-koeln.de/ ossk/ftpspace/deltavar. 


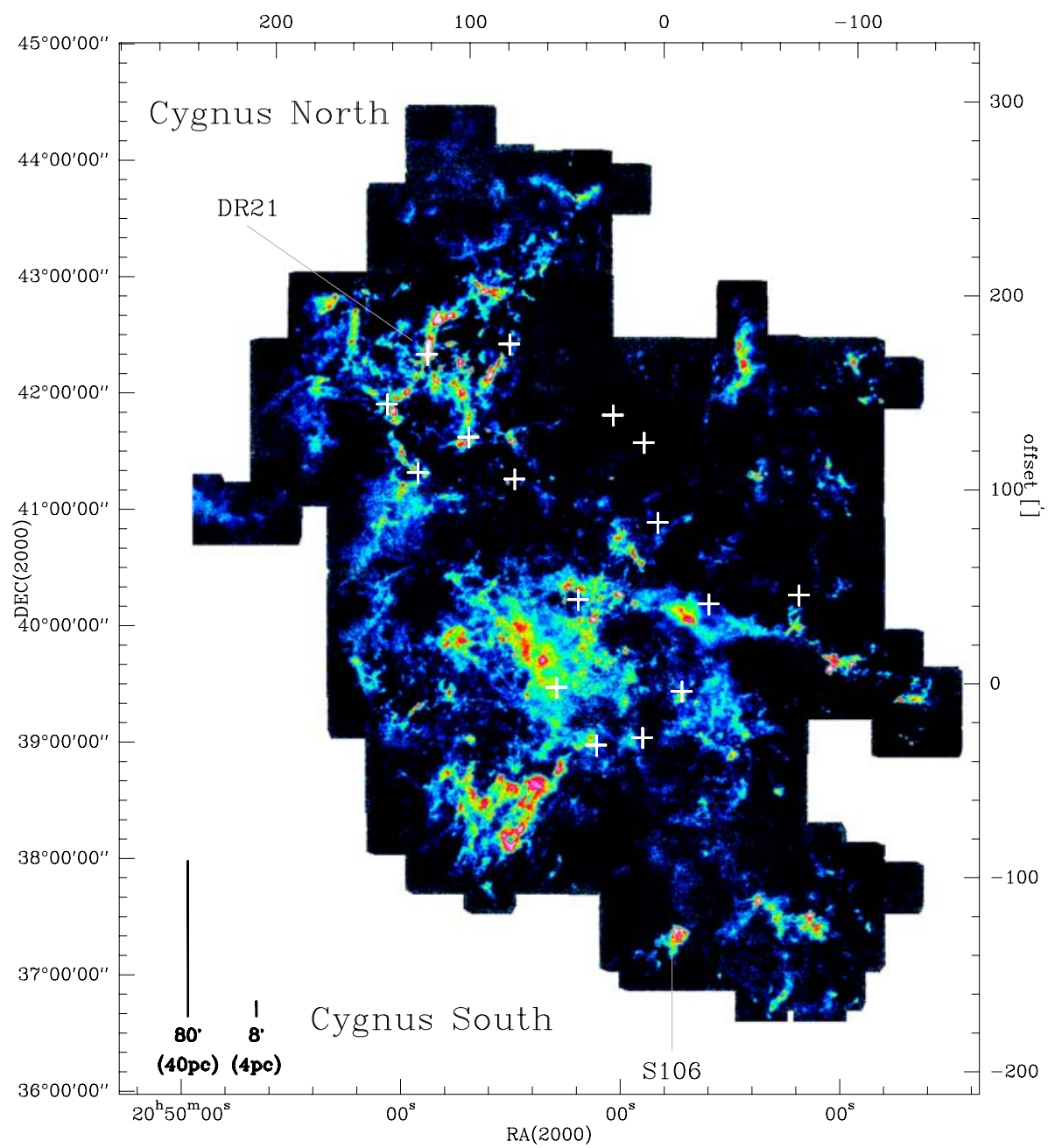

Fig. 1. Line-integrated map of Cygnus $\mathrm{X}$ in ${ }^{13} \mathrm{CO} 1 \rightarrow 0$ emission with the intensity $\left(-1\right.$ to $16 \mathrm{~K} \mathrm{~km} \mathrm{~s}^{-1}$ ) coded in color. Crosses indicate the location of thermal HII regions, the well-known star-forming regions DR21 and S106 are labeled.

$\Delta$-variance spectrum is dominated by the blurring from the finite telescope beam and radiometric noise.

\section{Results}

\subsection{FCRAO ${ }^{13} \mathrm{CO} 1 \rightarrow 0$ data}

Figure 1 shows the line intergrated map of Cygnus $\mathrm{X}$ in ${ }^{13} \mathrm{CO}$ $1 \rightarrow 0$ emission, tracing gas densities around $3 \times 10^{3} \mathrm{~cm}^{-3}$. The main features seen in Cygnus $\mathrm{X}$ are high-contrast filaments, mainly in the northern part of the map (DR21), and more diffuse emission with some higher density clouds in the south. Schneider et al. (2006) named these two regions "Cygnus North" and "Cygnus South". The molecular line emission from those clouds related to the Cygnus $X$ cloud complex covers a velocity range from roughly -15 to $+20 \mathrm{~km} \mathrm{~s}^{-1}$ (Schneider et al. 2006). However, some emission at velocities exceeding about $4 \mathrm{~km} \mathrm{~s}^{-1}$ (Piepenbrink \& Wendker 1988; Schneider et al. 2007) is caused by the "Great Cygnus Rift", a low-density gas feature at a distance of around $600 \mathrm{pc}$. It is responsible for a visual extinction of a few $A_{\mathrm{V}}$ (Dickel 1969; Schneider et al. 2007). In the figure, several arc or shell-like structures are visible, in particular in the northern part of the map where a cavity devoid of molecular gas (center at offsets 10',130') is surrounded by clouds that may have formed as swept-up material from the HII regions and the influence of the OB2 cluster. In Appendix $\mathrm{C}$ we show two additional figures, FCRAO ${ }^{13} \mathrm{CO} 1 \rightarrow 0$ data overlaid on a map of radio continuum emission at $1420 \mathrm{MHz}$ from the $\mathrm{CGPS}^{6}$ and on mid-IR emission from $\mathrm{MSX}^{7}$, in order to better depict the distribution of neutral/ionized and warm/cold gas.

In order to show the variation in the amount of structure as a function of length (lag) in the clouds, we apply the $\Delta$-variance method to the line integrated ${ }^{13} \mathrm{CO} 1 \rightarrow 0$ map. This is the first time that a map of $\sim 35$ square degrees is analyzed with the $\Delta$ variance. Typical map sizes in previous studies (Bensch et al. 2001; Sun et al. 2006) were in the range of 1 to 10 square degrees. We profited from the $(1 / \mathrm{rms})$ weighting feature implemented in the $\Delta$-variance algorithm developed by Ossenkopf et al. (2008a), so that areas with higher noise (in our case the map edges) contribute less to the $\Delta$-variance than areas with low, uniform noise. This improves the accuracy of the results with respect to distinguishing real signal from noise.

Figure 2 shows the $\Delta$-variance spectrum for the ${ }^{13} \mathrm{CO}$ $1 \rightarrow 0$ map. The error of each $\sigma_{\Delta}^{2}(L)$ is determined from the Poisson statistics. In Appendix D we explain the error calculation in more detail. The plot shows that with increasing lag $L$, the $\Delta$-variance first increases and reaches its peak value at $L_{\text {peak }}$, i.e. $8^{\prime}$ corresponding to $4 \mathrm{pc}$ for a distance of $1.7 \mathrm{kpc}$. With increasing lag, a second peak is observed at $80^{\prime}$ (40 pc) before

\footnotetext{
${ }^{6}$ Canadian galactic plane survey.

7 Midcourse space experiment.
} 


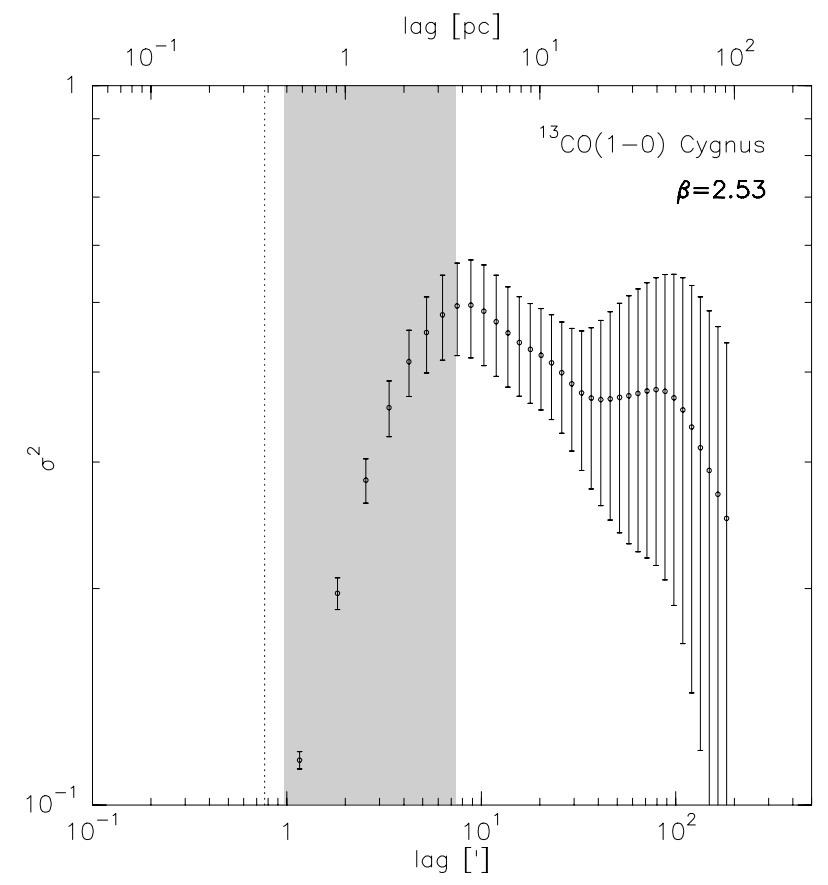

Fig. 2. The $\Delta$-variance spectrum of the line integrated map of the entire complex in the ${ }^{13} \mathrm{CO}$ emission. The slope $\beta$ is determined from a linear fit for data between lags $1^{\prime}$ and $8^{\prime}$ (indicated by the gray shaded area). The dotted line gives the beamsize $\left(45^{\prime \prime}\right)$.

the $\Delta$-variance decreases approximately with $L^{-2}$. Because this spectrum $^{8}$ exhibits well defined characteristic scales, it does not represent a fully self-similar structure, which would be the signpost of fully developed supersonic turbulence (Mac Low \& Ossenkopf 2000) and which would produce a pure power law.

The existence of characteristic scales in the $\Delta$-variance spectrum could be caused by (i) the true presence of preferred geometric scales in the system (length and width of filaments for example); (ii) decaying turbulence (Mac Low \& Ossenkopf 2000); (iii) energy injection from external or internal sources (largerscale accretion, spiral density waves, supernovae, outflows and winds, expanding HII regions, etc.); or by (iv) superposition of different clouds along the line-of-sight (note, however, that this effects does not create characteristic scales, but simply leads to several peaks in the $\Delta$-variance spectrum). It is therefore not straightforward to interpret the $\Delta$-variance spectrum of Cygnus since several of these effects may play a role.

We can, however, argue that the characteristic scales seen in the $\Delta$-variance spectrum are not caused by spurious line of sight effects. It is known that at least two groups of molecular clouds at distances of $600 \mathrm{pc}$ ("Great Rift") and $1.7 \mathrm{kpc}$ (see above) are found in the direction to Cygnus X. But it was shown and discussed in Schneider et al. (2007) that the column density of the Rift gas is very low $\left(A_{\mathrm{V}}<3^{m}\right)$ and not well traced by ${ }^{13} \mathrm{CO}$ emission. It is therefore well justified to assume that the whole $\Delta$-variance spectrum would be due to emission features arising from the clouds in $1.7 \mathrm{kpc}$ distance.

Characteristic scales caused by energy injection are plausible because this region is strongly influenced by radiation from the Cygnus OB2/OB9 clusters and a large number of outflow sources. The typical sizes of HII regions in the field (see Fig. C.1

\footnotetext{
${ }^{8}$ None of the scales corresponds to a structure introduced by the observational procedure because individual OTF-maps have a size of $10^{\prime} \times 20^{\prime}$.
}

in Appendix C) are on order of a few pc and larger, thus fitting to explain the origin of the observed scale of $4 \mathrm{pc}$. Outflows, however, act on much smaller scales. Nakamura \& Li (2007) report a characteristic scale of $0.3 \mathrm{pc}$ from their simulations of outflowdriven turbulence. This appears to be consistent with the scales observed in low-mass star-forming regions (e.g. as seen in velocity channel maps in NGC 1333, Quillen et al. 2005; Curtis et al. 2010). However, Cygnus $X$ forms a large number of highmass stars, which are expected to drive more energetic and most likely larger outflows compared to their model. A conclusive answer, therefore, requires more detailed numerical studies with a focus on high-mass star-forming regions. We also note that only a few signatures of SN-shells are found in Cygnus $\mathrm{X}$, and we conclude that the rate of SN-explosions in this area is very low (see overview in Reipurth \& Schneider 2008).

Another possible explanation for the features in the observed spectrum is that with ${ }^{13} \mathrm{CO}$ we indeed trace signatures of true morphological characteristics of the cloud. In concert with the visual inspection of the region (Fig. 1) it is appealing to identify the first peak around $4 \mathrm{pc}$ with small-scale filaments as also seen in many other nearby clouds (see Sect. 4.2.2). The second peak corresponds to the large-scale filamentary structure that dominates the global appearance Cygnus X.

However, there is another caveat. An alternative explanation to the $4 \mathrm{pc}$ scale could be that it is caused by radiative transfer effects in turbulent media (Padoan et al. 2000). As ${ }^{13} \mathrm{CO} 1 \rightarrow 0$ turns optically thick at column densities above about $N\left(\mathrm{H}_{2}\right)=$ $0.5-1 \times 10^{22} \mathrm{~cm}^{-2}$ (Ossenkopf 2002), this tracer is not capable of resolving any denser structure and all peaks are blurred out. The ${ }^{13} \mathrm{CO}$ observations may thus only provide a good reflection of the structure measured up to about $A_{\mathrm{V}}=5^{\mathrm{m}}$. The peak in the ${ }^{13} \mathrm{CO} \Delta$-variance basically shows the typcial size of the regions where the tracer turns optically thick, i.e. the ${ }^{13} \mathrm{CO}$ "photosphere" of the dense regions. The exact location of the peaks on the $\Delta$-variance spectrum gives some measure for the turbulence in the molecular cloud. They indicate a transition from more coherent structures, where the ${ }^{13} \mathrm{CO}$ emission falls into a narrow velocity interval, to a wide-spread turbulent distribution leading to a dilution of the optical depth in very broad lines. The details of this transition and its effects on the physical interpretation of the spectrum will be studied in a subsequent paper based on the $\Delta$-variance analysis of the velocity structure, i.e. studying individual velocity channel maps.

The power spectral index $\beta$ is typically determined for lags between the beamsize (indicated by a dashed line in Fig. 2) and the first significant structure component $\left(8^{\prime}\right)$. The value $\beta=2.53$ is at the lower end of typical values for molecular clouds (see, e.g., Bensch et al. (2001) for a compilation of clouds with indices between 2.5 and 3.3). This implies more structure on smaller scales and consequently that the lower column density gas has more structure. This was already pointed out by Falgarone et al. (2004), who studied the diffuse component of the interstellar medium, finding highly dynamic and fractal structures.

\subsection{Extinction maps}

\subsection{1. $\Delta$-variance from extinction maps}

Figures 3 to 6 show extinction maps of Galactic molecular clouds obtained with the method described in Appendix B and the corresponding $\Delta$-variance. For regions where ${ }^{13} \mathrm{CO}$ data were available from the literature, we additionally plotted the obtained $\Delta$-variance spectra. We determined the $\Delta$-variance without rms-weighting in the IDL routine "deltavar", using the 

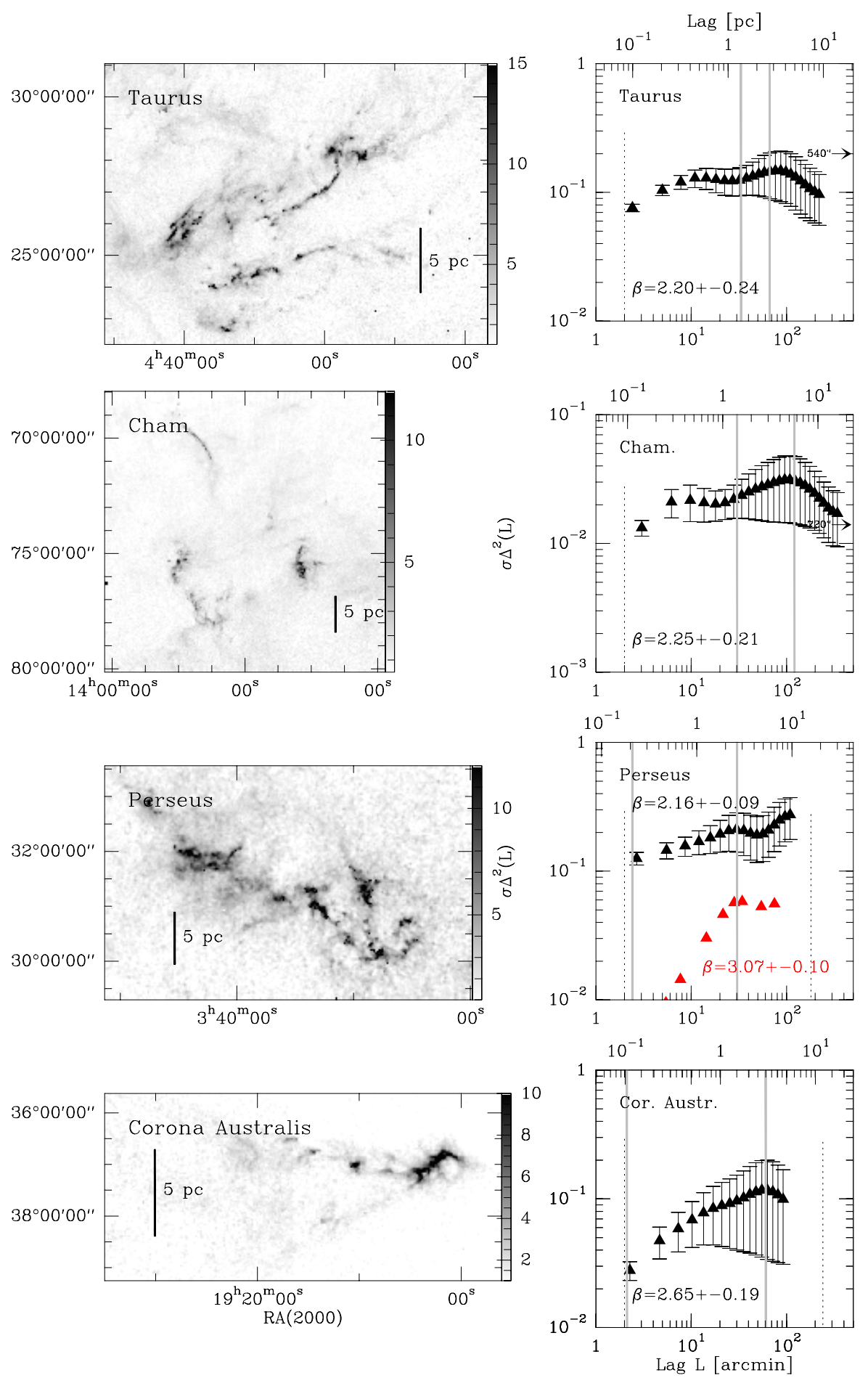

Fig. 3. Extinction maps of Galactic molecular clouds (left) and corresponding $\Delta$-variance spectrum with errors (right). See Appendix C how the error using Poisson statistic was calculated. The gray scale range of extinction $\left(A_{\mathrm{V}}\right)$ is given in the wedge of each plot. The dashed lines indicate the pixel size $\left(2^{\prime}\right)$ and the size of the map. If the mapsize is larger than the $x$-axis scale (500 arcmin), its size is noted in the plot. The small-scale slope $\beta$ and its error are given in each diagram, the fit range is indicated by two gray lines. The red curve for Perseus represents ${ }^{13} \mathrm{CO} 1 \rightarrow 0$ data from the Bell Labs (see Table 1 and Bensch et al. 2001). The lower $x$-axis is labeled in arcmin, the upper one in parsec. All coordinates are in RA and Dec epoch2000.

Mexican-hat filter with a diameter ratio of 1.5 , recommended by Ossenkopf et al. (2008a). All maps are extended and include all major emission regions (note that all $\Delta$-variance spectra cover lags well below the map extent). However, "empty" regions also contribute to the $\Delta$-variance and appear as large coherent structures so that they produce a decay of $\sigma_{\Delta}^{2}$ at large lags, which is shallower than the $L^{-2}$ decay that would represent a zero correlation at those scales (Ossenkopf et al. 2008a).

The regions that were analyzed comprise close-by (distance $<1 \mathrm{kpc}$ ), low-mass star-forming regions like Perseus, Taurus, or the Pipe Nebula, as well as more distant (up to $3 \mathrm{kpc}$ ) high-mass star-forming regions like Cygnus, Rosette, or W3. 
N. Schneider et al.: The link between molecular cloud structure and turbulence
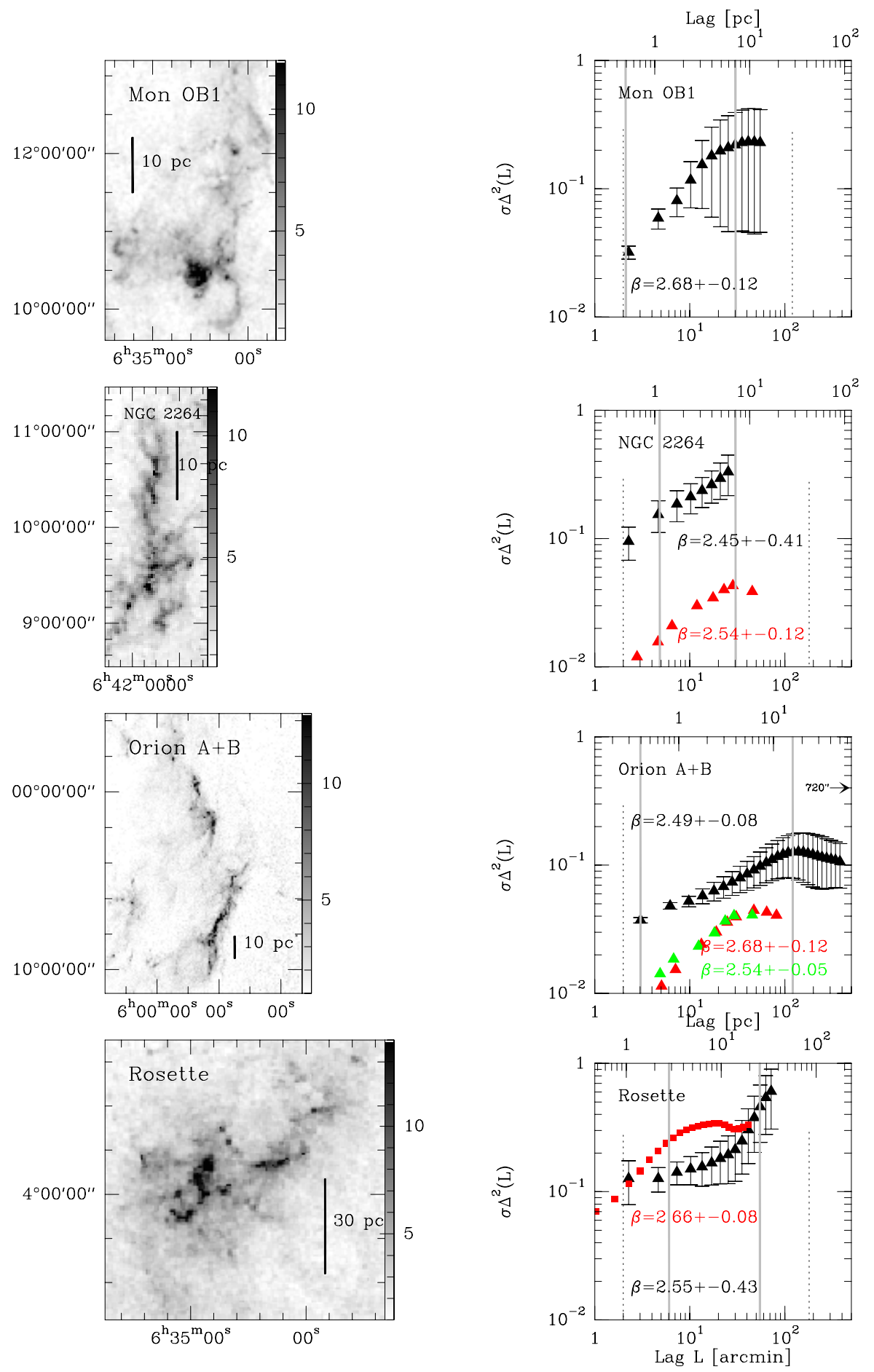

Fig. 4. Extinction maps of Galactic molecular clouds (left) and corresponding $\Delta$-variance (right). See Fig. 3 for further explanations. The red curves for NGC 2264 and Orion B represent ${ }^{13} \mathrm{CO} 1 \rightarrow 0$ data from Bell Labs (see Table 1 and Bench et al. 2001). The green curve shows the spectrum for Orion A using ${ }^{13} \mathrm{CO} 1 \rightarrow 0$ data from Bell Labs. The red curve for Rosette stems from ${ }^{13} \mathrm{CO} 1 \rightarrow 0$ data from FCRAO (Heyer et al. 2006).

A number of spectra show a simple rising curve (e.g. NGC 2264, Coalsack, Vela), some including a noise decrease plus a contribution from empty regions (W3, Taurus, Chameleon). The majority of spectra, however, have more complex shapes, (e.g. Lupus, MonR2, MonOB1, IC 5146, Pipe) with positive or negative curvature and/or two more or less pronounced peaks. Interestingly, the most filamentary structured clouds, partly with very thin, elongated features (Taurus, Chameleon) have the most complex double-peak curves and show low values of $\beta$.
It is tempting to interprete the two peaks in the $\Delta$-variance spectrum $(<1 \mathrm{pc}$ and $\sim 4 \mathrm{pc})$ in a purely geometrical view as the width and length of filaments. However, recent studies of the filamentary structure in the Aquila and Polaris regions with the Herschel satellite (André et al. 2010; Men'shchikov et al. 2010) obtained a much smaller typical width of the filaments. A detailed analysis of the radial profiles of the filaments observed with Herschel in Polaris, Aquila, and IC 5146 resulted in a characteristic filament width of about $0.1 \mathrm{pc}$ (Arzoumanian et al. (in prep.). The shape of the $\Delta$-variance and the value of 

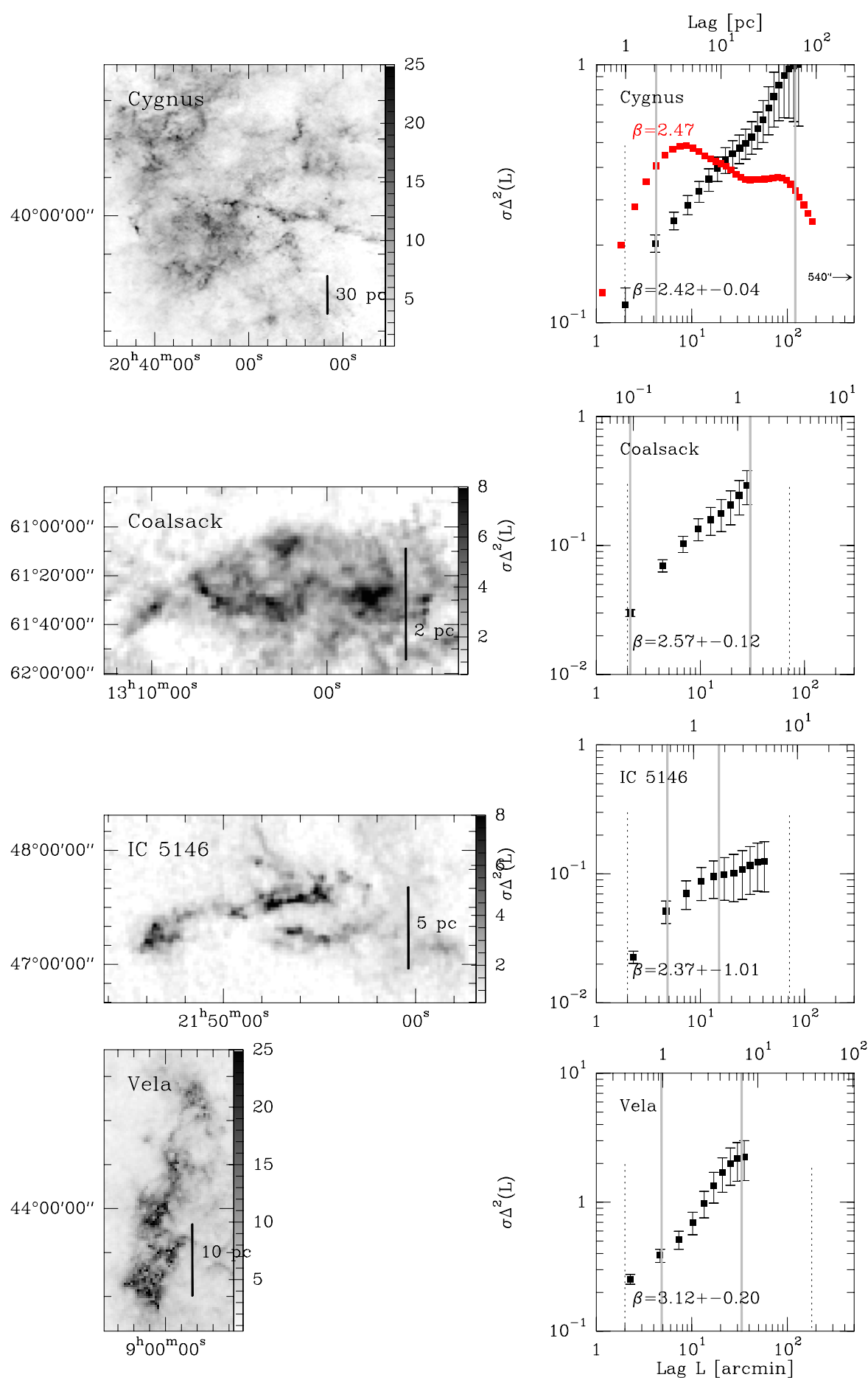

Fig. 5. Extinction maps of Galactic molecular clouds (left) and corresponding $\Delta$-variance (right). The red curve for Cygnus represents the ${ }^{13} \mathrm{CO}$ $1 \rightarrow 0$ data from the FCRAO survey. See Fig. 3 for further explanations.

$\beta$ weakly depend on the size of the selected region. Subregions in a map (e.g. for Vela, where only the high-column density region was selected from the $A_{\mathrm{V}}$ map), generally produce slightly steeper spectra with higher values of $\beta$. Large maps showing extended filamentary structures produce flatter spectra (e.g. Taurus, Chameleon, Pipe).

\subsubsection{Comparison to ${ }^{13} \mathrm{CO}$ data}

For regions where we had literature values or own data, we included the $\Delta$-variance determined from ${ }^{13} \mathrm{CO}$, i.e. Perseus,
NGC 2264, Orion A+B, Rosette, Cygnus, and Mon R2. The spectra of the massive GMCs Cygnus and Rosette look significantly different compared with the spectra derived from the extinction maps. For Cygnus, the curve determined from the $A_{\mathrm{V}^{-}}$ maps represents a fully self-similar scale distribution, covering all scales (since it is a very large and extended map), while the ${ }^{13} \mathrm{CO}$ spectrum shows the double-peak feature already discussed in Sect. 4.1. We attribute the two peaks from the ${ }^{13} \mathrm{CO}$ curve at $\sim 4$ and $\sim 40 \mathrm{pc}$ to the typical filament-sizes in Cygnus X, arising from clouds at a distance of $1.7 \mathrm{kpc}$ and/or optical depths effects of the ${ }^{13} \mathrm{CO}$ line emission. However, these two characteristic 

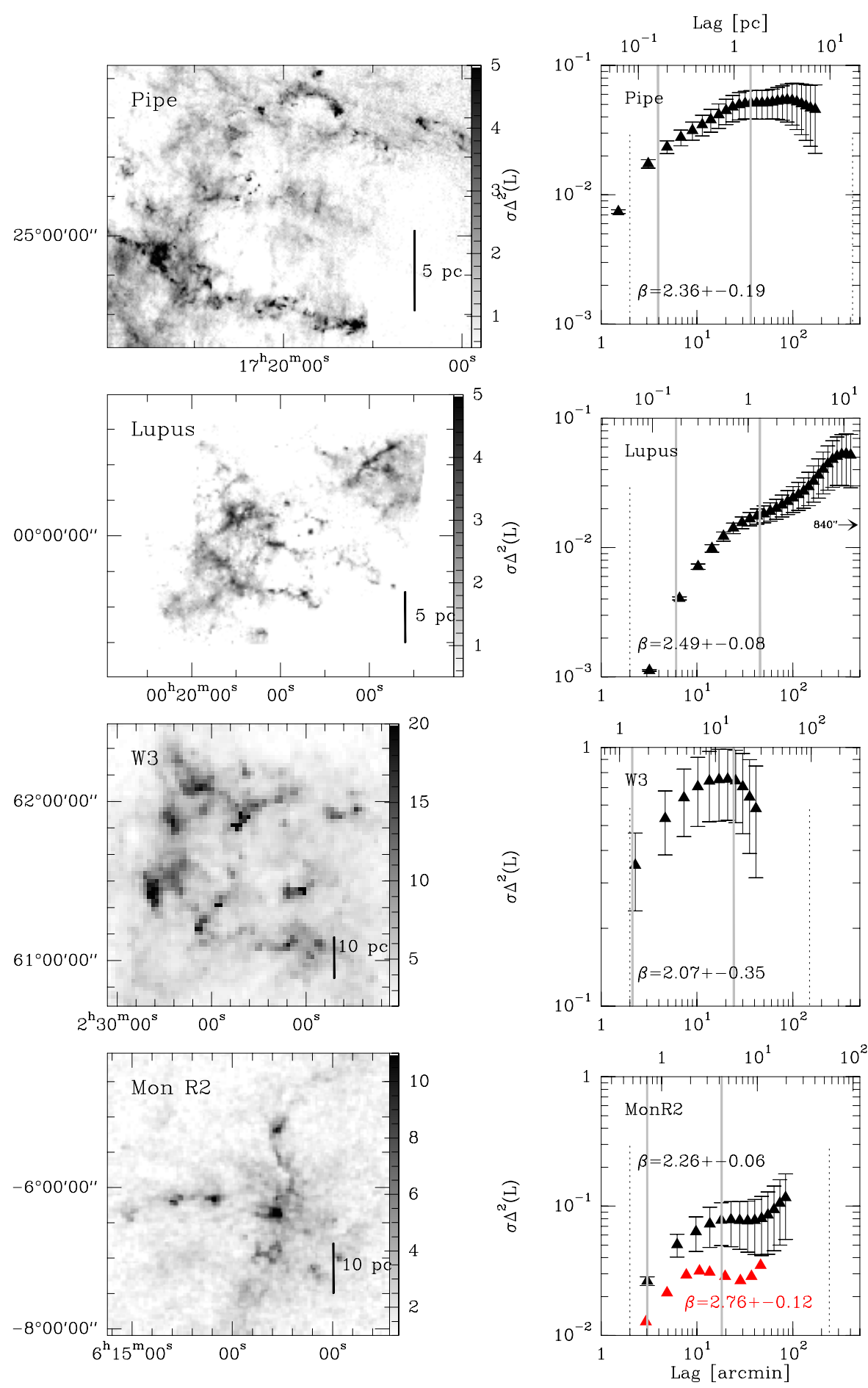

Fig. 6. Extinction maps of Galactic molecular clouds (left) and corresponding $\Delta$-variance (right). The red curve for MonR2 represents data from a ${ }^{13} \mathrm{CO} 1 \rightarrow 0$ Bell Labs survey (see Table 1 and Bench et al. 2001). See Fig. 3 for further explanations.

scales are not visible in the $\Delta$-variance spectrum of the extinction map. A possible explanation is that because the $A_{\mathrm{V}}$ map comprises all emission features along the line-of-sight, including the one from the low-density Cygnus Rift in $600 \mathrm{pc}$ distance, the resulting spectrum appears indeed self-similar because it is a composite of diffuse, fractal gas and denser structures. It is therefore possible that by using the ${ }^{13} \mathrm{CO}$ line, low-density larger scale structures that do not emit in ${ }^{13} \mathrm{CO}$ around $\sim 10^{\prime}-\sim 100^{\prime}$ are partly filtered out. However, the two spectra show a similar increase of the $\Delta$-variance (up to a scale of about $10^{\prime}$ ) with the slope of the extinction map being slightly shallower.
The Rosette spectrum has the same tendency to show a peaked structure in ${ }^{13} \mathrm{CO}$ with a plateau around $8 \mathrm{pc}$ and then a slight decrease, while the extinction map has no characteristic scale. However, for small lags, the spectra show a very different behavior. Because the ${ }^{13} \mathrm{CO}$ spectrum is not affected by noise for small lags, the spectrum increases in a similar way as for Cygnus (up to $20^{\prime}$ ). The spectrum from the $A_{\mathrm{V}}$-map, on the other hand, is limited by noise for small lags and is thus not directly comparable with the ${ }^{13} \mathrm{CO}$ spectrum. The opposite is true for large lags. The $8 \mathrm{pc}$ scale may again indicate the scale when the ${ }^{13} \mathrm{CO}$ line becomes optically thick. The larger scale for Rosette 


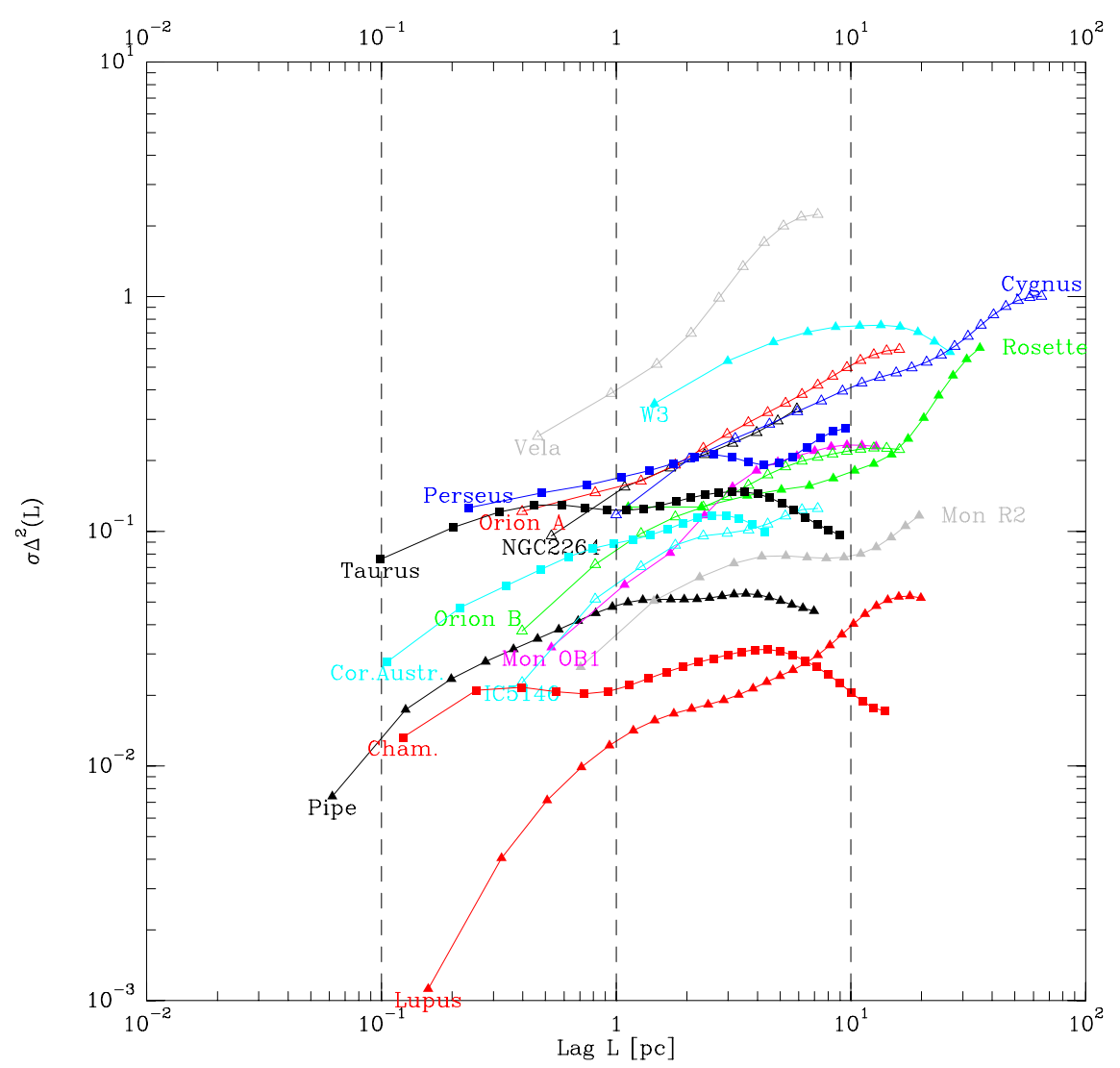

Fig. 7. $\Delta$-variance spectra of all sources where we obtained $A_{\mathrm{V}}$ maps in this study. The Lag scale is in parsec.

compared to Cygnus ( $4 \mathrm{pc}$ ) could arise because the average density of clumps in Cygnus (Schneider et al. 2006) is higher than in Rosette (Williams et al. 1995) and thus the ${ }^{13} \mathrm{CO}$ line is saturated on a smaller scale.

For the other sources (Perseus, Orion A/B, Mon R2, and NGC 2264), we obtained the $\Delta$-variance from Fig. 10 in Bensch et al. (2001), based on ${ }^{13} \mathrm{CO} 1 \rightarrow 0$ data from the Bell Labs $7 \mathrm{~m}$ telescope survey (Bally et al., priv. commun.). The form of the spectrum is rather similar to those obtained from the $A_{V}$-maps, but the location of peaks and the values of $\beta$ differ slightly. It seems that the angular resolution of the maps has an influence on the shape of the $\Delta$-variance because its form for the extinction maps (at $2^{\prime}$ resolution) corresponds well to the $1.7^{\prime}$ Bell Labs data but differs from that of the 50" resolution FCRAO surveys. However, Padoan et al. (2003) found for Taurus and Perseus that the structure function of ${ }^{13} \mathrm{CO}$ follows a power-law for linear scales between 0.3 and $3 \mathrm{pc}$, similar to our finding that a first characterestic scale is seen around $2.5 \mathrm{pc}$. A more recent study of Brunt (2010) of Taurus, comparing the power spectra determined from ${ }^{13} \mathrm{CO}$ and $A_{\mathrm{V}}$, showed that they are almost identical and that there is a break in the column density power spectrum around $1 \mathrm{pc}$. Below a wavenumber corresponding to a wavelength of $\sim 1 \mathrm{pc}$, Brunt (2010) found a power spectrum slope of 2.1 (similar to the delta variance slope found here). At wavenumbers above that (smaller spatial scales), a steeper spectrum was seen, with an index of 3.1. This power spectrum break is associated with anisotropy in the column density structure caused by repeated filaments (Hartmann 2002), which are possibly generated by gravitational collapse along magnetic field lines.

\subsubsection{The $\Delta$-variance for all clouds}

Figure 7 shows the $\Delta$-variance spectra for all clouds in this study obtained from the $A_{\mathrm{V}}$-maps. At small scales (below $1 \mathrm{pc}$ ) most sources show a non-constant spectral index steepening toward the resolution limit. This is consistent with decaying turbulence dissipated at small scales (Ossenkopf \& Mac Low 2002), but also with driven turbulence at small lags (Federrath et al. 2009). However, there are three exceptions: Chameleon and Taurus show an intermediate peak at $0.3-0.4 \mathrm{pc}$ and Perseus shows no clear indication of a steepening. The intermediate peak could mean that the extinction map is affected by a separate, more distant, component that is actually dominated by structures larger than assigned in the plot (see also discussion below). Alternatively, it could be produced by a systematic structure of the detected size that affects the turbulence in the cloud. Candidates for these structures are SN-shells. Expanding ionization fronts from $\mathrm{OB}$ associations impact the cloud structure as well. For example, it is known that Lupus is influenced by a subgroup of the Sco OB2 association (Tachihara et al. 2001) both by past $\mathrm{SN}$ explosions and present $\mathrm{OB}$ stars and their HII regions. Cygnus is exposed to the very massive OB2 cluster (Knödlseder et al. 2000), but lacks a significant number of SN shells. Orion $\mathrm{A}$ and $\mathrm{B}$ are influenced by stellar-wind driven compression centered on Ori OB 1b.

At scales above 1 pc nearly all low-mass SF clouds show a characteristic size scale as a peak of the $\Delta$-variance spectrum (see Sect. 4.2.4), i.e. Cor. Australis, Taurus, Perseus, Chameleon, Pipe show a common peak scale at $2.5-4.5 \mathrm{pc}$. This indicates the scale of the physical process governing the structure formation. This could e.g. be the scale at which a large-scale SN shock sweeping through the diffuse medium is broken at dense clouds, turning the systematic velocity into turbulence. The GMCs, on the other hand, show no break of the self-similar behavior at all up to the largest scales mapped. The Rosette is completely dominated by structure sizes close to the map size. At the largest, Galactic scales, energy injection due to, e.g., spiral 
N. Schneider et al.: The link between molecular cloud structure and turbulence

Table 1. Observed values of the power-law exponent $\beta$ (Col. 4) in different phases of the ISM (from diffuse HI gas to GMCs).

\begin{tabular}{|c|c|c|c|c|c|c|}
\hline Region & $\begin{array}{c}\text { Distance } \\
{[\mathrm{pc}]}\end{array}$ & Tracer & $\beta$ & $\begin{array}{c}\text { Peak 1 } \\
{[\mathrm{pc}]}\end{array}$ & $\begin{array}{c}\text { Peak 2 } \\
{[\mathrm{pc}]}\end{array}$ & Reference \\
\hline Diffuse HI & & $\mathrm{HI}$ & $2.6-2.8$ & & & Green et al. (1993) \\
\hline North Polar Spur & & HI & 3.6 & & & Miville-Deschenes (2003) \\
\hline \multicolumn{7}{|l|}{ Diffuse Clouds } \\
\hline Polaris Flare & 150 & ${ }^{13} \mathrm{CO} 1 \rightarrow 0$ & 2.77 & & & Stutzki et al. (1998) \\
\hline Polaris Flare & & ${ }^{13} \mathrm{CO} 2 \rightarrow 1$ & 2.76 & & & Falgarone et al. (1998), Stutzki et al. (1998) \\
\hline \multicolumn{7}{|c|}{ low-mass SF cloud } \\
\hline Perseus & 300 & ${ }^{\mathrm{T} 3} \mathrm{CO} 1 \rightarrow 0$ & 3.07 & 2.7 & $(>10)$ & Bensch et al. (2001) \\
\hline Perseus & & ${ }^{13} \mathrm{CO} 1 \rightarrow 0$ & 3.09 & & & Sun et al. (2006) \\
\hline Perseus & & ${ }^{13} \mathrm{CO} 2 \rightarrow 1$ & 3.03 & & & Sun et al. (2006) \\
\hline Perseus & & ${ }^{12} \mathrm{CO} 1 \rightarrow 0$ & 3.08 & & & Sun et al. (2006) \\
\hline Perseus & & ${ }^{12} \mathrm{CO} 3 \rightarrow 2$ & 3.15 & & & Sun et al. (2006) \\
\hline Perseus & & extinction & 2.55 & & & Sun et al. (2006) \\
\hline Perseus & & extinction & 2.16 & 2.5 & $(>10)$ & \\
\hline Taurus & 140 & extinction & 2.20 & 0.5 & 3.5 & \\
\hline Chameleon & 160 & extinction & 2.25 & 0.4 & 4.6 & \\
\hline Pipe Nebula & 140 & extinction & 2.36 & 1.5 & 3.8 & \\
\hline IC 5146 & 400 & extinction & 2.37 & (1.4) & $(>4.5)$ & \\
\hline Lupus & 100 & extinction & 2.49 & (1.5) & $(>10)$ & \\
\hline Coalsack & 150 & extinction & 2.57 & - & - & \\
\hline Corona Australis & 170 & extinction & 2.65 & - & 2.9 & \\
\hline \multicolumn{7}{|l|}{ GMC } \\
\hline W3 & 2200 & extinction & 2.07 & - & 11 & \\
\hline Cygnus & 1700 & ${ }^{13} \mathrm{CO} 1 \rightarrow 0$ & 2.47 & 4 & 40 & this study \\
\hline Cygnus & & extinction & 2.42 & - & - & \\
\hline Orion A & 450 & ${ }^{13} \mathrm{CO} 1 \rightarrow 0$ & 2.54 & - & 6 & Bensch et al. (2001) \\
\hline Orion A & & extinction & 2.49 & - & - & \\
\hline Orion B & 450 & ${ }^{13} \mathrm{CO} 1 \rightarrow 0$ & 2.68 & - & 6 & Bensch et al. (2001) \\
\hline Orion B & & extinction & 2.40 & - & $>13$ & \\
\hline Orion $\mathrm{A}+\mathrm{B}$ & & extinction & 2.49 & & 17 & \\
\hline Mon R2 & 800 & ${ }^{13} \mathrm{CO} 1 \rightarrow 0$ & 2.76 & 2.75 & $>11$ & Bensch et al. (2001) \\
\hline Mon R2 & & extinction & 2.26 & 3.9 & $>20$ & \\
\hline Mon OB1 & 800 & extinction & 2.55 & (4) & $>10$ & \\
\hline Rosette & 1600 & ${ }^{13} \mathrm{CO} 1 \rightarrow 0$ & 2.66 & - & $>7.5$ & Heyer et al. (2006) \\
\hline Rosette & & extinction & 2.55 & - & - & \\
\hline NGC 2264 & 800 & ${ }^{13} \mathrm{CO} 1 \rightarrow 0$ & 2.54 & - & 6.7 & Bensch et al. (2001) \\
\hline NGC 2264 & & extinction & 2.45 & - & - & \\
\hline Vela & 700 & extinction & 3.12 & - & $>7$ & \\
\hline
\end{tabular}

Notes. Values for $\beta$ obtained from our extinction maps are indicated in bold. Columns 5 and 6 indicate the size scale in parsec where spectra show peak values. Values in parenthesis are less reliable, the spectrum shows no prominent peak there. Values given only for peak 2 indicate that there is only one prominent peak. Note that for Orion we determined $\beta$ individually for the subregions Orion A and B (for direct comparison with the ${ }^{13} \mathrm{CO}$ data) and for the whole complex, which is also shown in Fig. 4.

density waves should be visible on a kpc size, which is well above our limits.

\subsubsection{Are there characteristic size scales depending on the type of cloud?}

Although the $\Delta$-variance determined from extinction maps shows interesting particularities for different clouds, one has to note that there can be large differences in the value of $\beta$ within each individual complex, as was shown by Sun et al. (2006) for Perseus. We thus have to be careful in interpretating different values of $\beta$ and the shape of the spectrum depending on the type of cloud and tracer. In Table 1 we listed $\beta$ for different gas phases, from diffuse HI gas to low-and high-mass starforming regions, obtained from maps with different tracers (HI, isotopomeric $\mathrm{CO}$ lines, extinction maps and dust continuum). In total, the values range from 2.07 (W3) to 3.12 (Vela) and are thus in the typical range of values determined for other studies (e.g. Bensch et al. 2001). However, if we exclude the most extreme data points for W3 and Vela and the very dispersed results for Perseus, the range of $\beta$-values is much more narrow, only 2.16 to 2.76 . But in any case, no systematic trend can be seen between the value of $\beta$ and the type of cloud/gas phase. However, all $\beta$ values determined from ${ }^{13} \mathrm{CO}$ are systematically higher than those obtained from the extinction maps. In addition, based only on ${ }^{13} \mathrm{CO}$ data, there is a slight tendency that high-mass SF regions have lower values of $\beta$ than low-mass SF regions. A similar trend is reported by Kainulainen et al. (2009) in the column density PDF, which deviates from the log-normal form and develops power-law tails in massive SF regions. We do not see a trend with distance (since shorter distances to a cloud resolve smaller scale structures and thus $\beta$ should be higher).

We also investigated the presence of a characteristic scale in the spectra with one or two peaks, i.e. we listed in Table 1 the parsec-scale where a first and possible second peak were observed. Interestingly, all clouds that show a double-peak structure are low-mass SF regions in galactic regions that are not (much) affected by line-of-sight crowding. The more massive 
and denser high-mass star-forming clouds show no clear doublepeak structure. This can partly be because most of them are farther away (W3, Cygnus, Rosette are all at distances $>1.5 \mathrm{kpc}$ ) and thus the extinction maps are sensitive for all gas along the line-of-sight and therefore to all structure scales. However, other massive clouds like NGC 2264, the Orion clouds, and Vela are closer $(<1 \mathrm{kpc})$ and do not show a double-peak structure either. The first peak for low-mass SF clouds occurs at size scales that are characteristic for molcular clumps, i.e. 0.4 to $1.5 \mathrm{pc}$ (with one higher value for Perseus of $2.5 \mathrm{pc}$ ). The second peak is found between 2.9 and 4.6 pc. Blitz \& Williams (1997) found a characteristic size scale of $0.25-0.5 \mathrm{pc}$, indicating a change in structure in Taurus, conform with our finding for Taurus. A small sample of clouds (Orion A, $\rho$ Oph, Taurus, and L1512) was investigated by Falgarone et al. (2004) using ${ }^{13} \mathrm{CO} 1 \rightarrow 0$ emission. They found a typical size scale of around $0.5 \mathrm{pc}$ for the width and 1 to $18 \mathrm{pc}$ for the lengths of filaments. Our sample is more homogeneous in its values, and we may be tracing two different physical processes here, one leading to the formation of clumps $(<1 \mathrm{pc}$ scale) and one to the formation of filamentary structures (4 pc scale as the lengths of the filaments). However, the typical width of filaments is $<1 \mathrm{pc}$ as well, which complicates the picture. These scales may therefore be associated with turbulence driven on small scales and turbulence driven on larger scales. This is an intersting result because the physical properties and the driving vary from cloud to cloud. It might be, for example, that energy injection from outflows is more important for Perseus than for Taurus (the latter contains much fewer sources) and thus leads to a slightly different characterstic scale since turbulence driven by outflows acts on smaller scales than turbulence driven by large scale events like SN explosions. However, different modes of turbulence forcing can also lead to significantly different $\Delta$-variance spectra (Federrrath et al. 2009) and turbulence statistics in the ISM (Federrath et al. 2010), and the same is true for strongly self-gravitating systems (Klessen 2000; Ossenkopf et al. 2001; Kainulainen et al. 2009). The picture may be different for massive GMCs that show no characteristic scales in $A_{V}$ maps and none in ${ }^{13} \mathrm{CO}$ maps, if we assume that the characteristic scales around a few parsec arise from optical depths effects. Heyer et al. (2006) for example found no difference in the scaling coefficent for the velocity structure function (see, e.g., Brunt 2003, for details) for the Rosette Molecular Cloud, an active GMC forming massive stars and strongly influenced by an expanding HII region, as well as G216-2.5, an even more massive cloud, but with very little low-mass SF. They conclude that energy input at large scales sustains the global turbulence in molecular clouds while effects like expanding HII regions or outflows act locally in modifying the turbulent structure.

It should also be noted that our resolution is not high enough to resolve molecular cores. The typical size of massive dense cores in high-mass star-forming regions is $0.1 \mathrm{pc}$ (e.g. Motte et al. 2007), and they typically show sub-fragmentation (Bontemps et al. 2010), so that they are not cores in the sense of single star-forming units. For low-mass SF regions, such as rho Ophiuchi for example, the typical size of a pre-stellar core is well below the 0.1 pc scale (e.g. Motte et al. 1998). In any case, our resolution is above $0.1 \mathrm{pc}$. It would be interesting to apply the $\Delta$-variance on large maps at high angular resolution to resolve cores, clumps, and filaments at the same time. This will be possible with the large-scale imaging at far-infrared wavelengths (70 to $500 \mu \mathrm{m})$ of low-and high-mass star-forming regions performed with the Herschel satellite (André et al. 2010; Motte et al. 2010; Molinari et al. 2010), which provides an angular resolution between $6^{\prime \prime}$ and $40^{\prime \prime}$.

\section{3. $\Delta$-variance and turbulence models}

To arrive at a better physical understanding of interstellar turbulence and gain deeper insight into the processes that govern the formation and evolution of molecular clouds it is important to compare the observational data with theoretical and numerical models of turbulent flows. This is useful, because models with different parameters (purely hydrodynamic or with magnetic field, with or without self-gravity, driven or decaying turbulence, different driving sources, etc.) will produce different $\Delta$-variance spectra and accordingly may allow us to distinguish between different theories. For example, the $\Delta$-variance approach was used by Mac Low \& Ossenkopf (2000) to characterize the density and the velocity structure of interstellar turbulence simulations. They showed that driven, supersonic hydrodynamic turbulence (with or without magnetic field) can maintain a well-defined, selfsimilar behavior at scales below the driving scale. Klessen et al. (2000), and Heitsch et al. (2001), and Ossenkopf et al. (2008b) showed that energy injection on a particular scale does not create density enhancements on that scale, but on one which is $20 \%$ to $25 \%$ smaller. Finding pure power-law $\Delta$-variance spectra in molecular cloud data therefore indicates that the observed turbulence is driven from the outside. We note that virtually all theoretical models that applied the $\Delta$-variance are based on Gaussian random fields or fractal Brownian motion and yield slopes that are well within the observed range $(\approx 2.3-3.1$, see Table 1$)$. It is possible that different physical large-scale driving mechanisms (spiral density waves, supernovae, expanding HII regions) may lead to different spectral behavior.

On the other hand, there are various processes that can imprint a characteristic scale on a molecular cloud structure. For example, it was shown by Mac Low \& Ossenkopf (2000) that freely decaying turbulence causes a change of the slope, i.e. a deviation from self-similarity. The only distinctive scale is at the lower end, i.e. at the diffusion scale. A similar result was reported by Nakamura \& Li (2007), who studied the effects of explosive partially collimated outflows on a background of decaying turbulence and found a noticeable break in the velocity power spectrum at the outflow scale. Ossenkopf et al. (2001) focused on self-gravity and demonstrated that self-gravity introduces a characteristic scale to the spectrum which evolves in time as more and more gas is accumulated in dense cores. This effect is probably only visible when using optically thin tracers sensitive for dense gas (Ossenkopf 2002), which is not the case for our extinction maps. However, the observational data are not fully conclusive. While Ossenkopf et al. (2001) find a negative slope in Serpens (Testi et al. 1998), a more recent study performed by Ossenkopf et al. (2008b) based on a large mmcontinuum map in $\rho$ Oph (Motte et al. 1998) did not confirm this and instead reported the typical shape of the $\Delta$-variance as discussed above.

Another recent application of the $\Delta$-variance on model simulations was performed by Federrath, Klessen, and Schmidt (2009). They used the $\Delta$-variance to characterize the density structure produced in supersonic, isothermal hydrodynamic models with two limiting cases of turbulence forcing: solenoidal (divergence-free) vs. compressive (curl-free) forcing. Table 2 shows that the compressive forcing models produce systematically higher values of $\beta$ than the solenoidal ones. All values are, however, at the upper limit of the range typically obtained from molecular cloud studies. Interestingly, a high value of $\beta$ is better consistent with the $\Delta$-variance spectra determined from diffuse Galactic HI emission (see Table 1), and seems consistent with Kolmogorov scaling of incompressible turbulence 
Table 2. Values of $\beta$ from hydrodynamic simulations of supersonic, isothermal turbulence with solenodial (divergence-free) and compressive (curl-free) forcing (Federrath et al. 2009).

\begin{tabular}{lll}
\hline \hline & Forcing & $\beta$ \\
\hline 3d-hydrodynamic simulation & solenoidal & 2.89 \\
3d-hydrodynamic simulation & compressive & 3.44 \\
2d-projection & solenoidal & 2.81 \\
2d-projection & compressive & 3.37 \\
\hline
\end{tabular}

(Falgarone et al. 2004). The transition to incompressible turbulence, however, is expected to occur close to the sonic scale at about 0.1 pc (Vázquez-Semadeni et al. 2003; Federrath et al. 2010). Scales larger than that are clearly in the supersonic regime. The steepening of the power-law index $\beta$ toward large scales in HI clouds and in the numerical model with compressive forcing is consequently unlikely to represent incompressible turbulence. It is more likely that the cloud-like structures on intermediate scales (see Fig. 1, right panel in Federrath et al. 2009) produce a relative steepening of the spectral index on large scales. A similar effect may occur for dense molecular cloud structures embedded in the diffuse atomic HI gas. In contrast, the steepening of $\beta$ on scales smaller than $0.1 \mathrm{pc}$ (see Bensch et al. 2001) indicates a transition to coherent cores (Goodman et al. 1998) with transonic to subsonic, almost incompressible turbulence, because this transition is likely to occur close to the sonic scale (Vazquez-Semadeni et al. 2003; Federrath et al. 2010; Pineda et al. 2010).

\section{Summary}

We presented a 35 square degrees ${ }^{13} \mathrm{CO} 1 \rightarrow 0$ molecular line survey of Cygnus $\mathrm{X}$, taken with the FCRAO, and visual extinction $\left(A_{\mathrm{V}}\right)$ maps of 17 Galactic clouds, obtained from near-IR 2MASS data, in order to analyze the spatial structure of molecular clouds with the $\Delta$-variance method.

For Cygnus, we found no single characteristic scale in the $A_{\mathrm{V}}$-map at all. A double-peak spectrum of the $\Delta$-variance was revealed by ${ }^{13} \mathrm{CO} 1 \rightarrow 0$ data with peaks at $4 \mathrm{pc}$ and $40 \mathrm{pc}$. The $4 \mathrm{pc}$ scale corresponds to the typical length of small filaments ubiquitously found in low-mass star-forming nearby clouds, while the second peak around $40 \mathrm{pc}$ arises from the largest filamentary structures seen in Cygnus. This scale is below the whole extent of the cloud $(\sim 100 \mathrm{pc})$. An alternative explanation is that the $4 \mathrm{pc}$ scale is the characteristic scale when the ${ }^{13} \mathrm{CO} 1 \rightarrow 0$ line becomes optically thick (typically up to an extinction of about $A_{\mathrm{V}}=5^{\mathrm{m}}$ ). Thus, ${ }^{13} \mathrm{CO}$ observations may generally provide a good reflection of the structure measured only up to this $A_{\mathrm{V}}=5^{m}$ limit. The exact location of the peaks on the $\Delta$-variance spectrum gives some measure for the turbulence in the molecular cloud. They indicate a transition from more coherent structures, where the ${ }^{13} \mathrm{CO}$ emission falls into a narrow velocity interval, to a wide-spread turbulent distribution leading to a dilution of the optical depth in very broad lines. Applying the $\Delta$-variance on velocity channel maps of molecular line data will help to better understand this point. The origin of energy injection in Cygnus can be manifold. It is known that the region is strongly affected by radiation from the Cygnus OB2/OB9 clusters, expanding HII regions, a large number of outflow sources, and at least one supernova-remnant.

The power spectral index $\beta$ for Cygnus, determined from the $\Delta$-variance spectrum $\sigma_{\Delta}^{2} \propto L^{\beta-2}$ with the size parameter $L$, has a value of $\beta=2.53$, which is at the lower end of typical values for molecular clouds (typically between 2.5 and 3.3).

The $\Delta$-variance spectra obtained from the $A_{\mathrm{V}}$ maps show differences between low-mass star-forming clouds and massive giant molecular clouds in terms of shape of the spectrum and values of the slope $\beta$. Many of the low-mass SF clouds have a double-peak structure with characteristic size scales around $1 \mathrm{pc}$ and $4 \mathrm{pc}$. The physical process governing structure formation could be the scale at which a large-scale SN shock or expanding HII regions sweeping through the diffuse medium are broken at dense clouds, which turns the systematic velocity into turbulence. Giant molecular clouds show no characteristic scale in the $A_{\mathrm{V}}$-maps, which can be ascribed partly to a distance effect owing to a larger line-of-sight (LOS) confusion. The values of $\beta$ show no clear trends, although there is a tendency that all $\beta$ values determined from ${ }^{13} \mathrm{CO}$ are systematically higher than those obtained from the extinction maps and that high-mass SF regions have lower values of $\beta$ than low-mass SF regions. We do not see a trend with distance, although this might have been expected because shorter distances to a cloud resolve smaller scale structures and consquently $\beta$ should be higher. A comparison between the $\Delta$-variance determined from model simulations (supersonic, isothermal hydrodynamic models with solenoidal (divergence-free) vs. compressive (curl-free) forcing by Federrath et al. (2009) and observations shows that the model values are systematically higher, but still consistent with the observed range of $\Delta$-variance slopes across different clouds.

Overall, this study shows the full complexity of cloud structure analysis, giving some indications of physics studied with the various methods. However, it leaves many open questions, which clearly require more work in comparing observations and turbulence models in the context of different methods.

Acknowledgements. We thank M. Heyer and J. Williams for providing us with the ${ }^{13} \mathrm{CO} 1 \rightarrow 0$ FCRAO data from the Rosette Molecular Cloud.

R.S.K. and C.F. acknowledge financial support from the German Bundesministerium für Bildung und Forschung via the ASTRONET project STAR FORMAT (grant 05A09VHA) and from the Deutsche Forschungsgemeinschaft (DFG) under grants no. KL 1358/1, KL 1358/4, KL 1359/5, KL 1358/10, and KL 1358/11. R.S.K. furthermore thanks for subsidies from a Frontier grant of Heidelberg University sponsored by the German Excellence Initiative and for support from the Landesstiftung Baden-Württemberg via their program International Collaboration II (grant P-LS-SPII/18). R.S.K. also thanks the KIPAC at Stanford University and the Department of Astronomy and Astrophysics at the University of California at Santa Cruz for their warm hospitality during a sabbatical stay in spring 2010. A part of this work was supported by the French Agence National de la Recherche (ANR) project PROBes No. 08-blanc-0241.

\section{Appendix A: Data quality}

The data points of the surveys were irregularly sampled and thus regridded to a fully sampled 22 '. 5 grid. The aliased noise power was minimized and the full resolution of the telescope retained. The convolution kernel used was

$$
\frac{J_{1}(2 \pi a x)}{2 \pi a x} \frac{J_{1}\left(c x / R_{\max }\right)}{c x / R_{\max }} \mathrm{e}^{-(2 b x)^{2}} \Pi\left(R_{\max }\right)
$$

with $J_{1}$ the first-order Bessel function, $x$ the distance from the observed data point, $R_{\max }$ the truncation radius $\left(R_{\max }=3\right), a=$ $0.9009, b=0.21505$, and $c=3.831706$. $\Pi\left(R_{\max }\right)$ is used as a pill box function with $\Pi=1$ for $R<R_{\max }$ and $\Pi=0$ for $R>R_{\max }$.

The gridded spectra were converted and stored in CLASS spectra files. CLASS is a part of the GILDAS package and was used to further reduce the data. The data for small regions, typically $30^{\prime} \times 30^{\prime}$, were reduced in a data reduction pipeline. First, 


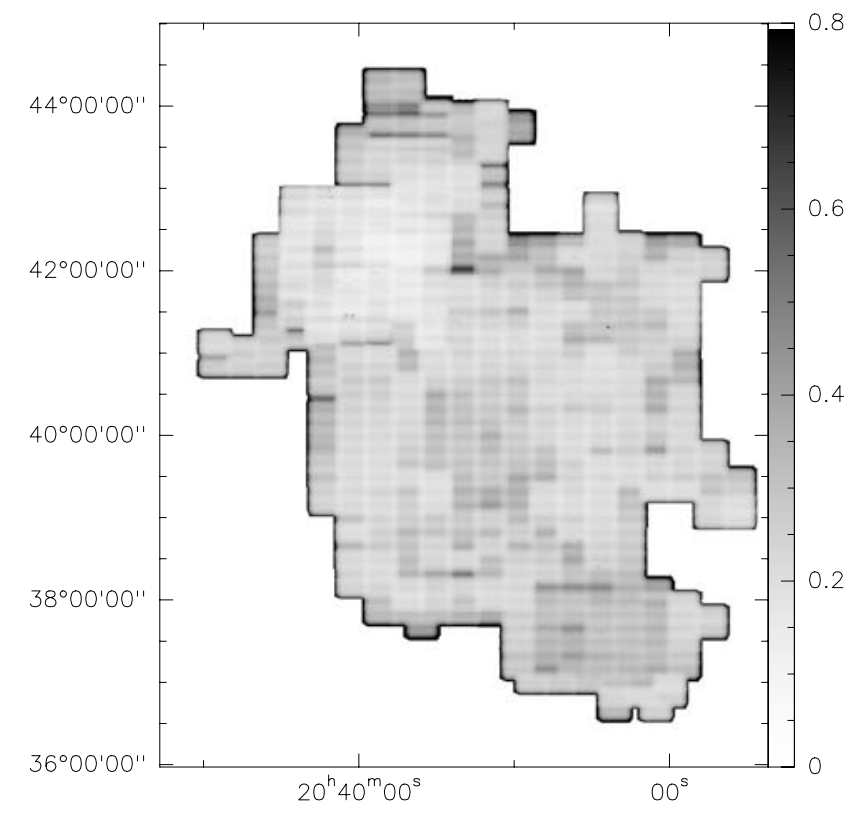

Fig. A.1. $\mathrm{Rms}$ map of the ${ }^{13} \mathrm{CO}\left(\mathrm{C}^{18} \mathrm{O}\right)$ data in $\mathrm{K}$.

all spectra in the region were averaged to determine the window around the emission to be excluded from the baseline fit. Second, a first to third order baseline was subtracted from all spectra. The reduced spectra were then written in $3 d$ data cubes (RA, Dec, velocity). Afterwards cubes of the individual regions were merged into a single $3 \mathrm{~d}$ data cube for application of the $\Delta$-variance.

In order to quantify the quality of the survey, we produced rms noise maps of all line tracers, the one of ${ }^{13} \mathrm{CO}$ is shown in Fig. A.1. Obviously the noise is not uniform, but shows a checkerboard structure and the edges are more noisy than the center. Both effects are owing to the observing mode. In the OTF mode, a single position is passed by several pixels of the receiver array, resulting in a dense sampling and thus, an improved signal-to-noise ratio in the inner regions of an OTF map. Toward the map edges, the sampling is not as dense, causing higher rms values. Because the individual maps were observed with overlap to assemble the final large scale data set, the overlap regions are sampled a little more densely. The lower rms values in these overlaps create the checkerboard structure. The plot also highlights the areas that were observed twice, namely the DR21 region.

\section{Appendix B: Extinction maps from dust reddening of 2MASS sources: the example of Cygnus $X$}

The main purpose of developing AvMAP instead of using other programs (e.g. NICER; Lombardi et al. 2001) is that it can handle foreground stars up to larger distances and without assumptions or off-field evaluation of the density of these foreground stars. In this way, we are able to image the most distant clouds. To illustrate our technique of deriving extinction maps from near-IR 2MASS data we describe in detail as an example the case of Cygnus $\mathrm{X}$.

The resulting $A_{\mathrm{V}}$ map for a field of $10^{\circ} \times 10^{\circ}$ toward Cygnus $\mathrm{X}$ is displayed in Fig. B.1. In this large field, a total of $4.5 \times 10^{6}$ stars are found with an average of 12.4 stars per $\operatorname{arcmin}^{2}$. The individual $A_{\mathrm{V}}$ are measured as the quadratic, uncertainty weighted average of the two $A_{\mathrm{V}}$ estimates obtained

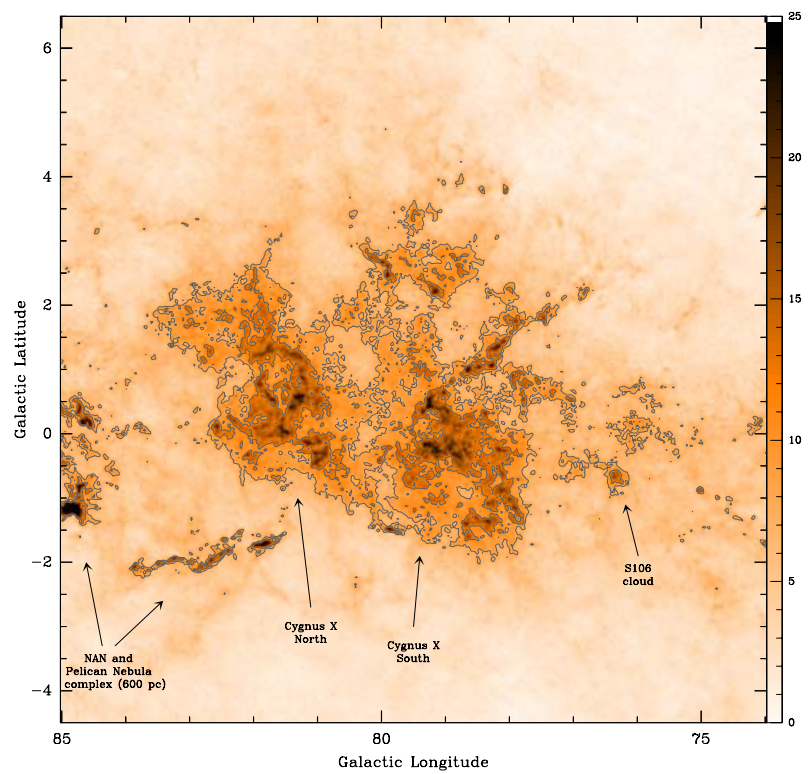

Fig. B.1. Map of the extinction $\left(A_{\mathrm{V}}\right)$ toward the Cygnus $\mathrm{X}$ region obtained from the reddening of $2 \mathrm{MASS}$ point sources. The $A_{\mathrm{V}}$ derivation is optimized for a distance of $1.7 \mathrm{kpc}$, and is over-estimated for the more nearby complex of the North America and Pelican Nebulae. The Cygnus $\mathrm{X}$ complex is well visible and appears as mostly two large complexes referred to in Schneider et al. (2006) as Cygnus X North and South. One can also note that the S106 cloud is well visible in the southern part of Cygnus X.

from the two 2MASS colors $[J-H]$ and $[H-K]$. The individual uncertainties are given by the quadratic sum of photometric uncertainties (from the catalog) and of the uncertainties on the intrinsic colors of a typical Galactic star (see for instance Lombardi et al. 2001, 2010, for more details). The adopted values are $[J-H]_{0}=0.45 \pm 0.15$, and $[H-K]_{0}=0.12 \pm 0.05$ with the associated uncertainties measured from the color dispersions for a population of Galactic stars as measured using simulations with the Besançon Galactic models (Robin et al. 2003; see below).

\section{B.1. Prediction of the density of foreground stars}

The above described method to estimate dust column densities entirely relies on the hypothesis that the near-IR sources are background stars. However, for a distant region like Cygnus X, a significant density of foreground stars is expected. To properly measure the dust column density, the foreground stars have to be filtered out (see detailed discussion in Cambrésy et al. 2002).

We use the predictions of the Besançon stellar models to derive a systematic, independent estimate of the expected number of foreground $\left(n_{\mathrm{fg}}\right)$ stars for any Galactic direction. Catalogs of sources were simulated using the online simulator (http://model .obs-besancon. fr/) for a list of representative Galactic directions. For each simulated catalog, the number of stars brighter than the 2MASS completeness levels for all filters $(J=17.0, H=16.5$ and $K=16.0)$, and at a distance smaller than the cloud distance $(d=1.7 \mathrm{kpc})$ is calculated to derive the expected density of foreground stars. Figure B.2 displays the resulting values of $n_{\mathrm{fg}}$ for 20 positions on the Galactic plane $(l a t=0)$ from lon $=10$ to $90^{\circ}$, and for 21 other positions at three fixed longitudes (lat $=75^{\circ}, 80$ and $85^{\circ}$ ) from lat $=0$ to $10^{\circ}$. Note that $n_{\mathrm{fg}}$ is expressed as a function of the Galactocentric distance $r_{\mathrm{GC}}$ (down axis) using $R_{\mathrm{GC}}^{\odot}=8.5 \mathrm{kpc}$. The Cygnus $\mathrm{X}$ 


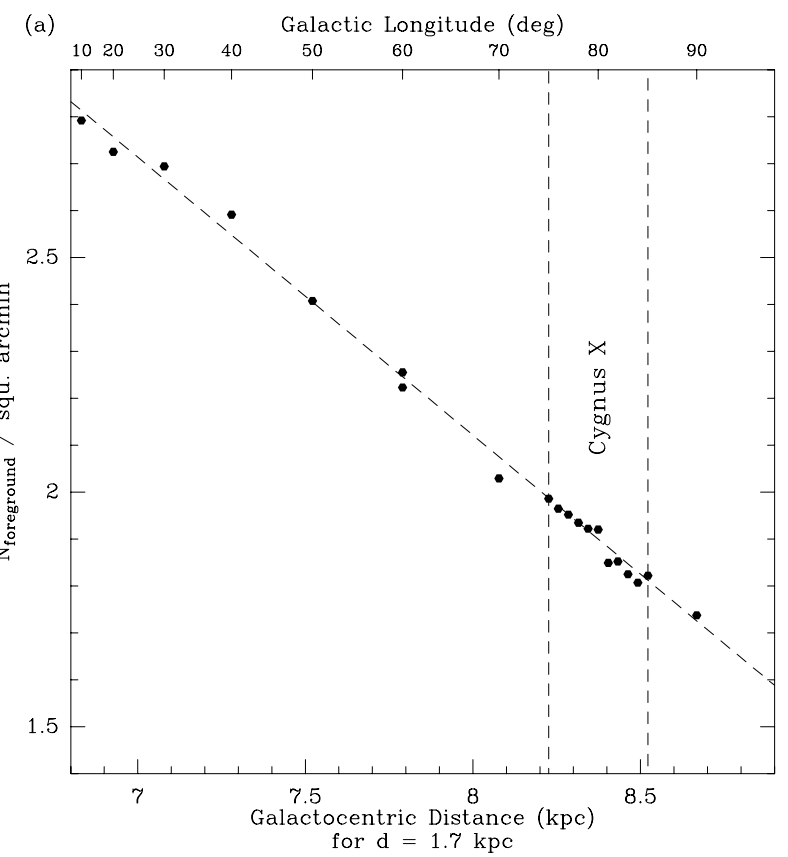

(b)

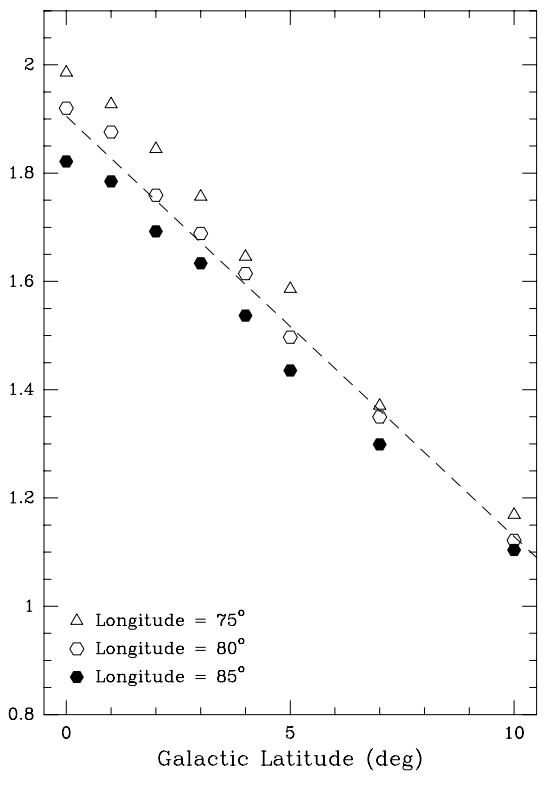

Fig. B.2. Variation of $n_{\mathrm{fg}}$ measured from simulations using the Besançon model (see text) a) in the Galactic plane as a function of longitude; and b) in the Cygnus $\mathrm{X}$ region as a function of latitude. The variation with longitude is actually expressed as a function with $r_{\mathrm{GC}}$ (see text). These predicted foreground star densities have been derived for a distance from Sun of $1.7 \mathrm{kpc}$. The adopted linear interpolations are displayed as dashed lines.

region corresponds to $r_{\mathrm{GC}}$ between 8.22 and $8.52 \mathrm{kpc}$ where $n_{\mathrm{fg}}$ is decreasing from $\sim 2$ to 1.8 star per $\operatorname{arcmin}^{2}$, which is on the order of $10 \%$ of the average density of 2MASS sources. The effect of latitude is larger with a typical decrease from $~ 1.9$ to 1.1 star per $\operatorname{arcmin}^{2}$ for lat $=10^{\circ}$. In order to filter out foreground stars, we then assumed that they are always the bluest stars in each direction, which may not be always fully correct for the lowest extinction regions.

\section{B.2. Distribution of young stars}

Young stars are usually more luminous than the population of background stars. If they are low or intermediate mass young stars, they are still above the main sequence, and if they are massive, they are luminous and necessarily young due to their fast stellar evolution. In the 2MASS catalog, these young stars are not necessarily brighter than the surroundings because of the flux attenuation (extinction) of the parental cloud. However, following the same idea like to derive the average total extinction, the fluxes of all 2MASS sources can be de-reddened to evaluate their intrinsic brightness. Because the young stars form in clusters, maps of the distribution of the brightest IR sources after dereddening should trace well the embedded clusters. We thus use the same $A_{\mathrm{V}}$-mapping procedure to produce at the same time density maps of the brightest dereddened stars. For the flux cut to be used, a good compromise between the 2MASS sensitivity and the typical brightness of young stars was found to be between $K=12$ and $K=13$. With a cut at $K=12$ a large number of cluster members can be found. In contrast, a cut at $K=13$ is more selective and can secure the detection of a cluster difficult to recognize.

Figure B. 3 below displays the map of the density of stars with $K>13$ in the Cygnus $\mathrm{X}$ region after dereddening. It clearly shows a large-scale distribution of stars in the Galactic plane with several extended, higher concentration, of stars. On the top

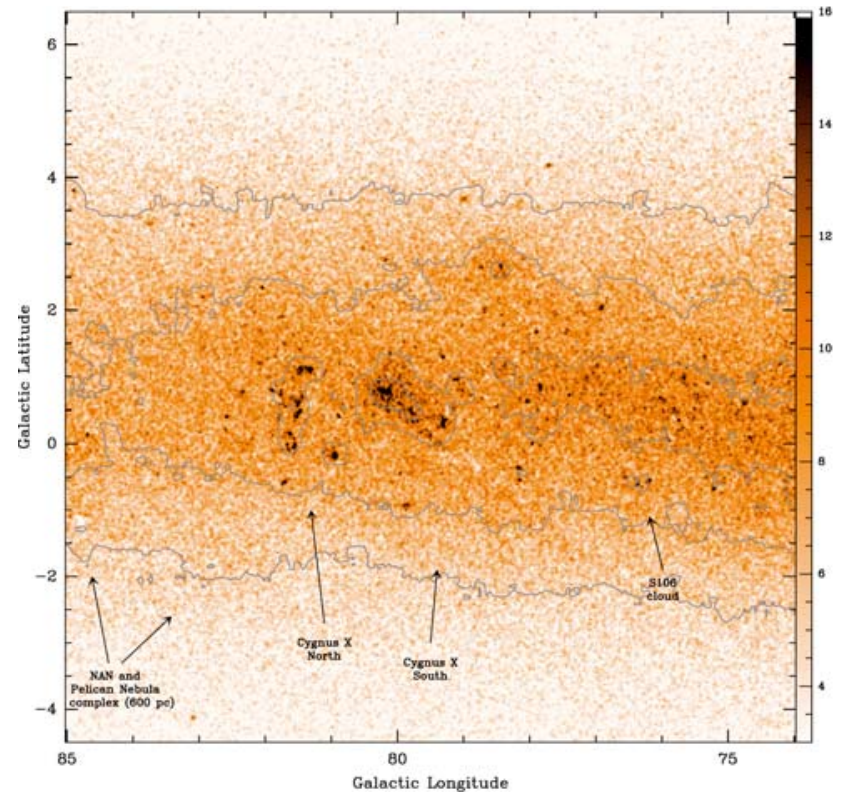

Fig. B.3. Map of the source density with a dereddened $K$ band $(2 \mu \mathrm{m})$ magnitude lower than 13. The linear scale is given as a slider on the right and is expressed in number of sources per arcmin ${ }^{2}$. The Cyg-OB2 association in the middle is clearly visible (see also Knödlseder 2000). A number of embedded clusters inside Cygnus X North could be the seeds for a newly formed $\mathrm{OB}$ association.

of this large-scale distribution a number of small-scale clusters are apparent. A fraction of these clusters were also recognized by eye inspection of the 2MASS images by Dutra \& Bica (2001), and using a simplified version of the present imaging procedure by LeDuigou \& Knödlseder (2002). 


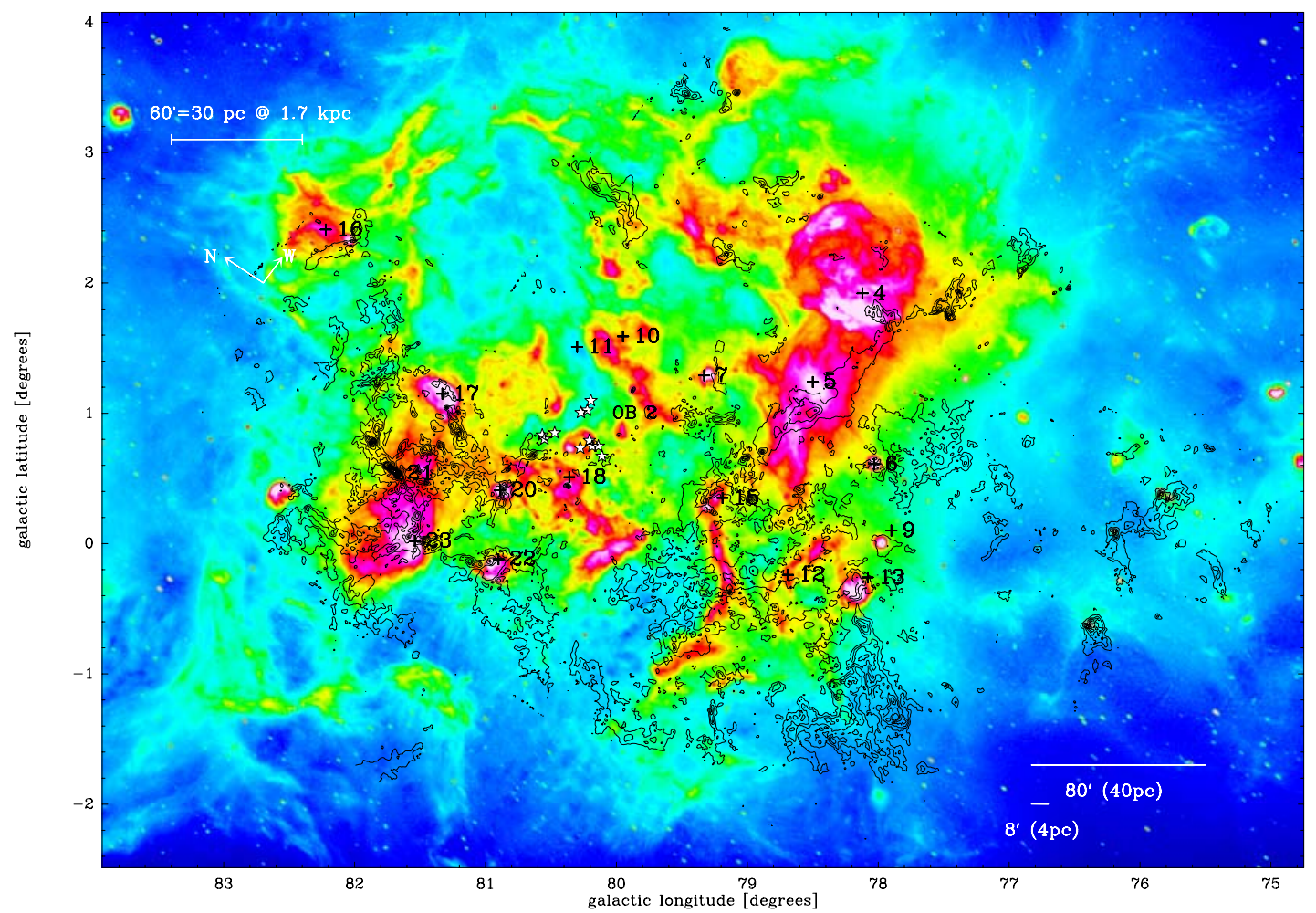

Fig. C.1. The Cygnus X region in color scale at radio wavelengths (1420 MHz) from the Canadian galactic plane survey. Contours outline the ${ }^{13} \mathrm{CO} 1 \rightarrow 0$ emission from the FCRAO. The stars indicate the most massive members of the Cyg OB2 association and crosses with numbers the HII regions DR4 to DR23.

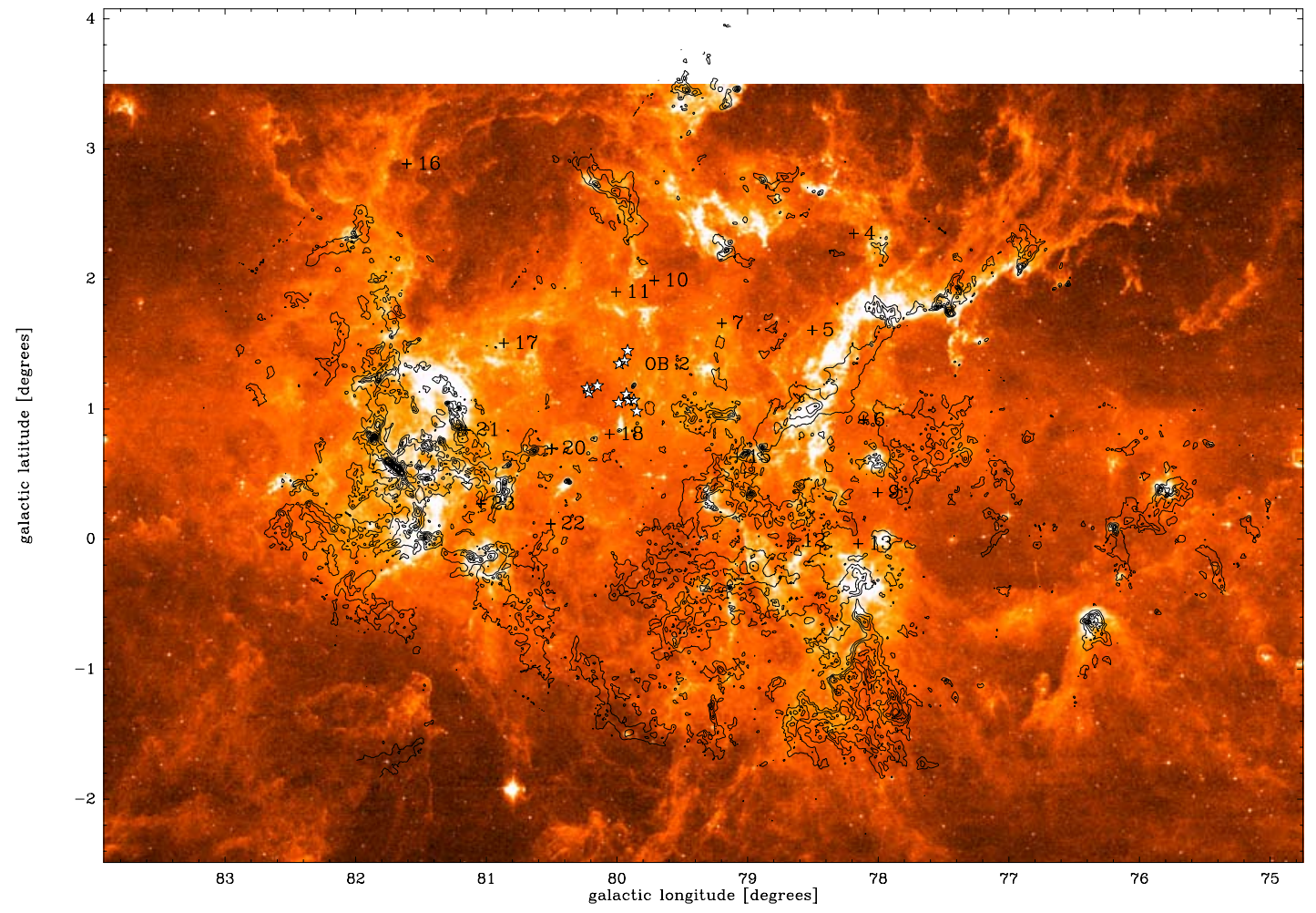

Fig. C.2. The Cygnus X region as seen at $8 \mu \mathrm{m}$ by the MSX, again with FCRAO ${ }^{13} \mathrm{CO} 1 \rightarrow 0$ emission overlaid as black contours.

\section{B.3. "AvMAP", the fortran implementation of the method}

In practice here for the Cygnus $\mathrm{X}$ field displayed in Figs. B.1 and B.3, the visual extinction $A_{\mathrm{V}}$ was evaluated by averaging individual $A_{\mathrm{V}}$ for stars inside Gaussian beams for each position in the map and after excluding the required number of probable foreground sources. We adopted a grid of $450 \times 450$ pixels with $80^{\prime \prime}$ spacing, and a resulting Gaussian resolution of 1.9' (Nyqvist sampling). The typical number of 2MASS sources per 
beam typically varies from 15 to 25 , but goes down to close to 10 in regions of high extinction, and can even reach the typical number of foreground sources ( 4 to 5 per beam) for the darkest regions. This is particulary the case for the region at lon $\sim 84.7^{\circ}$, which corresponds to the North America and Pelican Nebulae, which are known to be at a distance of only $800 \mathrm{pc}$ and for which, therefore, the number of foreground stars removed is too large and leads to an over-estimate of $A_{\mathrm{V}}$.

In order to compute the different steps to derive $A_{\mathrm{V}}$ and the density of young stars in each pixel of large maps, we coded a FORTRAN90 program with an inner algorithm that is optimized to reduce the number of accesses to the (large) catalog of sources. Indeed, for any large map, several millions of stars have to be processed with their coordinates, fluxes, and uncertainties. It is impossible to go through millions of sources for each pixel when typically $10^{5}$ pixels are required to image the field. Instead, for each step of the calculation, each source is read only once, and it is the contribution of this source to the final result which is calculated. Altogether, three paths/steps are required to compute $A_{\mathrm{V}}$ and the density of sources $n_{\mathrm{K}}$.

The most recent version of AvMAP produces images directly in FITS format using the astrolib package. The best supported projection method is gnomonic even if some others implemented projection may work fine too (less tested).

\section{Appendix C: Figures of radio continuum and mid-IR emission of Cygnus $X$}

Figures C.1 and C.2 show an overlay of ${ }^{13} \mathrm{CO} 1 \rightarrow 0$ emission from FCRAO on a radio continuum survey at $1420 \mathrm{MHz}$ obtained from the CGPS $^{9}$ and on mid-IR emission at $8 \mu \mathrm{m}$ from the MSX-satellite, respectively. The known thermal HII-regions from the Downes \& Rinehart survey (1966) are indicated. Because the mid-IR emission traces warm PAHs (Polycyclic Aromatic Hydrocarbonates), there is a very good correlation to the radio continuum of the HII regions that serve as the heating sources for the dust. The molecular line emission is partly correlated with the mid-IR/radio continuum emission (bright-rimmed borders of the cloud like for S106), but in other regions uncorrelated (for example the emission seen at lower galactic latitudes). Examples for a stellar-wind/radiation driven bubble sre known for by NGC $6913\left(l=77^{\circ}, b=0.5^{\circ}\right)$ (also Schneider et al. 2007) and around the main $\mathrm{OB}$ cluster in Cygnus X (OB 2, $l=80.2^{\circ}$, $b=1^{\circ}$ ). The nicely defined bubble at $l=78.2^{\circ}, b=2.1^{\circ}$ is the well-known SNR gamma Cygni.

\section{Appendix D: Error calculation}

To test the significance of the $\Delta$-variance, in particular the interpretation of the Poisson error bars on the interpretation of the spectra, we performed some numerical experiments to assess the actual impact of the "counting error" that is quantified in terms of the Poisson error bars. The counting error is due to the limited sampling of an overall (considered as infinite) structure by a finite observation. We mimick this situation by selecting different parts of a larger periodic structure.

Figure D.1 shows two different test maps, one fractal Brownian motion (fBm) structure and one periodic structure, a chess board. (See Ossenkopf et al. (2008a), for more details.) The $\Delta$-variance was determined from the whole map and from three regions with different sizes and is displayed in the lower

\footnotetext{
9 http://www1.cadc-ccda.hia-iha.nrc-cnrc.gc.ca/cgps
}

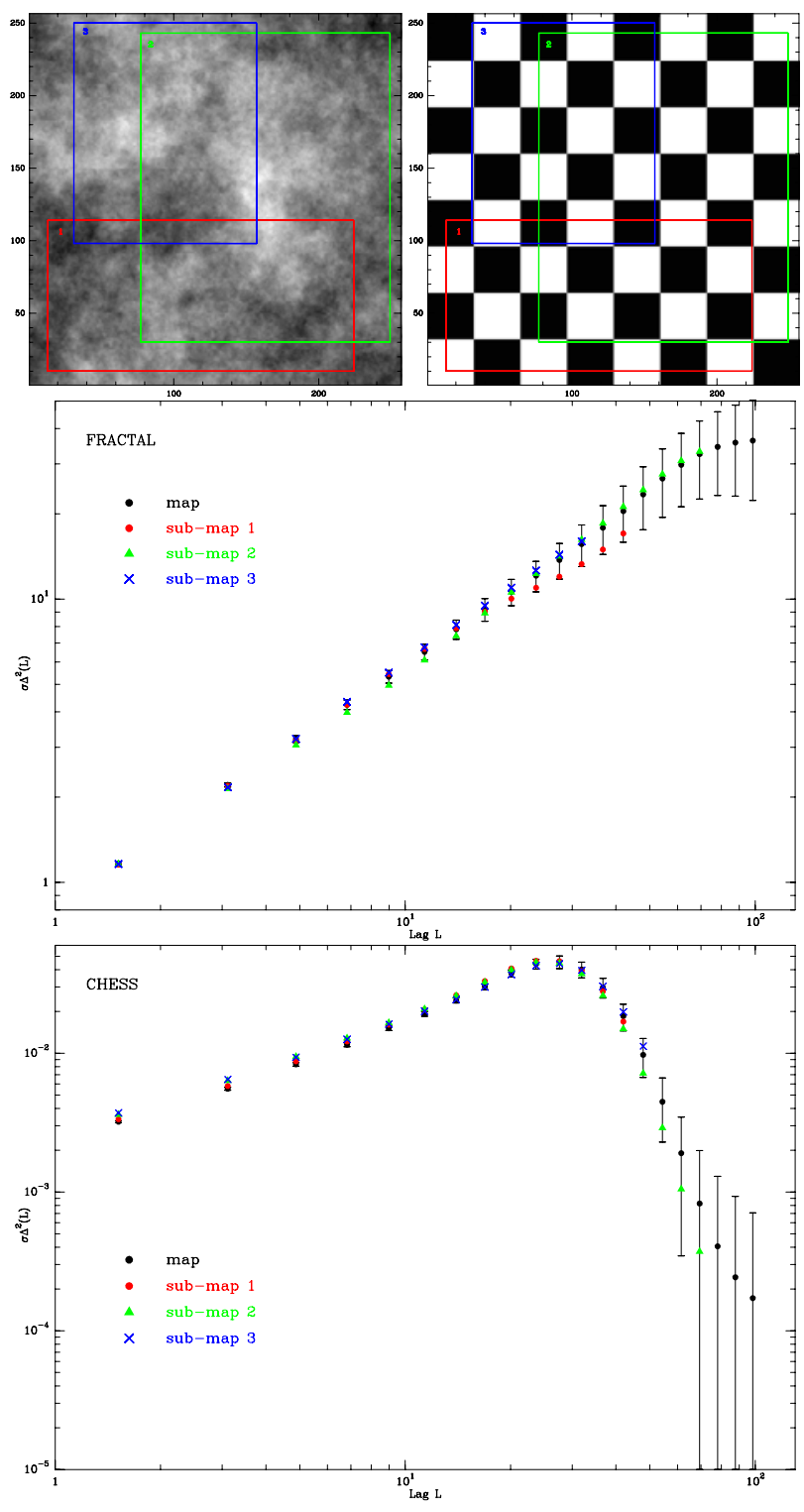

Fig. D.1. Upper panels left: fBm-structure right: chess board. The three regions from which the $\Delta$-variance (lower panels) was determined are indicated in the plot.

panels. Obviously, that when observing different subregions of a larger structure, the $\Delta$-variance spectrum of the substructure covers the full range of values indicated by the Poisson error bars, i.e. the application of the Poisson statistics for the computation of the uncertainty is confirmed. However, we find that the $\Delta$-variance values at neighboring lags are not independent, but are strongly correlated. The different view on the large structure "bends" the whole $\Delta$-variance spectrum upward or downward, mainly at large lags, but it hardly introduces mutual variations between the $\Delta$-variance values for similar lags. Consequently, we think that all the discussed structure in the $\Delta$-variance spectra, including primary and secondary peaks, are significant because the error bars only describe the total uncertainty of the spectrum as a whole, but not variations of the $\Delta$-variance between neighboring lags. 


\section{References}

Allan, D. W. 1966, Proc. IEEE, 54, 221

André, P., Men'shchikov, A., Bontemps, S., et al. 2010, A\&A, 518, L102 (special issue Herschel)

Ballesteros-Paredes, J., \& Mac Low, M.-M. 2002, ApJ, 570, 734

Ballesteros-Paredes, J., Hartmann, L., Vazquez-Semadeni, E., Heitsch, F., \& Zamora-Aviles, M. A. 2011, MNRAS, 411, 65

Banerjee, R., Klessen, R. S., \& Fendt, C. 2007, ApJ, 668, 1028

Banerjee, R., Vázquez-Semadeni, E., Hennebelle, P., \& Klessen, R. S. 2009, MNRAS, 389, 1082

Bate, M. 2009, MNRAS, 392, 1363

Bensch, F., Stutzki, J., \& Ossenkopf, V. 2001, A\&A, 266, 636

Blitz, L., \& Williams, J. P. 1997, ApJ, 488, L145

Bontemps, S., Motte, F., Csengeri, T., \& Schneider, N. 2010, A\&A, 524, A18

Brunt, C. M. 2003, ApJ, 583, 280

Brunt, C. M. 2010, A\&A, 513, A67

Brunt, C. M., \& Heyer, M. 2002a, ApJ, 566, 276

Brunt, C. M., \& Heyer, M. 2002b, ApJ, 566, 289

Brunt, C. M., Heyer, M. H., \& Mac Low, M.-M. 2009, A\&A, 504, 883

Cambrésy, L., Beichman, C. A., Jarrett, T. H., \& Cutri, R. M. 2002, AJ, 123, 2559

Curtis, E. I., Richer, J. S., Swift, J. J., \& Williams, J. P. 2010, MNRAS, 408, 1516

Dib, S., Brandenburg, A., Kim, J., et al. 2008, ApJ, 678, 105

Dib, S., Walcher, J. C., Heyer, M., et al. 2009, MNRAS, 398, 1201

Dickel, H. R., Wendker, H. J., \& Bierritz, J. H. 1969, A\&A, 1, 270

Downes, D., \& Rinehart, R. 1966, ApJ, 144, 937

Dutra, C. M., \& Bica, E. 2001, A\&A, 376, 434

Elmegreen, B., \& Falgarone, E. 1996, ApJ, 471, 816

Elmegreen, B. G., \& Scalo, J. 2004, ARA\&A, 42, 211

Erickson, N. R., Grosslein, R. M., Erickson, R. B., \& Weinreb, S. 1999, IEEE, 47, 2212

Falgarone, E., Philipps, T. G., \& Walker, C. K. 1991, ApJ, 378, 186

Falgarone, E., Hily-Blant, P., \& Levrier, F. 2004, Astrophys. Space Sci., 292, 89

Federrath, C., Klessen, R. S., \& Schmidt, W. 2008, ApJ, 688, L79

Federrath, C., Klessen, R. S., \& Schmidt, W. 2009, ApJ, 692, 364

Federrath, C., Duval, J., Klessen, R. S., Schmidt, W., \& Mac Low, M.-M. 2010, A\&A, 512, A81

Goldreich, P., \& Kwan, J. 1974, ApJ, 189, 441

Goldsmith, P. F., Snell, R. L., Hasegawa, T., \& Ukita, N. 1987, ApJ, 314, 525

Goodman, A. A., Barranco, J. A., Wilner, D. J., et al. 1998, ApJ, 504, 223

Gritschneder, M., Naab, T., Walch, S., Burkert, A., \& Heitsch, F. 2009, ApJ, 694, L26

Hartmann, L. 2002, ApJ, 578, 914

Heitsch, F., Mac Low, M.-M, \& Klessen, R. S. 2001, ApJ, 547, 280

Heitsch, F., Hartmann, L. W., Slyz, A. D., et al. 2008, ApJ, 674, 316

Hennebelle, P., \& Audit, E. 2007, A\&A, 465, 431

Hennebelle, P., Audit, E., \& Miville-Deschenes, M.-A. 2007, A\&A, 465, 445

Hennebelle, P., Banerjee, R., Vázquez-Semadeni, E., Klessen, R. S., \& Audit, E. 2008, A\&A, 486, L43

Hennebelle, P., Commercon, B., Joos, M., Klessen, R. S., et al. 2011, A\&A, 528, A72

Heyer, M., \& Schloerb 1997, ApJ, 475, 173

Heyer, M., \& Brunt, C. 2004, ApJ, 615, L45

Heyer, M. H., Williams, J. P., \& Brunt, C. M. 2006, ApJ, 643, 956

Heyer, M., Krawczyk, C., Duval, J., \& Jackson, J. M. 2009, ApJ, 699, 1092

Kainulainen, J., Beuther, H., Henning, T., \& Plume, R. 2009, A\&A, 508, L35

Klessen, R. S. 2000, ApJ, 535, 869

Klessen, R. S., Heitsch, F., \& Mac Low, M.-M. 2000, ApJ, 535, 887

Klessen, R. S., \& Hennebelle, P. 2010, A\&A, 520, A17

Knödlseder, J. 2000, A\&A, 360, 539

Kolmogorov, A. 1941, Dokl. Akad. Nauk SSSR, 30, 301

Kramer, C., Stutzki, J., Röhrig, R., \& Corneliussen, U. 1998, A\&A, 329, 249

Krumholz, M. 2006, ApJ, 641, L45

Krumholz, M. R,, Matzner, C. D., \& McKee, C. F. 2006, ApJ, 566, 302

Krumholz, M., Klein, R. I., \& McKee, C. F. 2007, ApJ, 656, 959

Krumholz, M. R., \& Matzner, C. D. 2009, ApJ, 703, 1352

Lada, C. J., Lada, E. A., Clemens, D. P., \& Bally, J. 1994, ApJ, 429, 694

Larson, R. B. 1981, MNRAS, 194, 806

LeDuigou, J.-M., \& Knödlseder, J. 2002, A\&A, 392, 869

Li, Z.-Y., \& Nakamura, F. 2006, ApJ, 640, L187
Lombardi, M., \& Alves, J. 2001, A\&A, 377, 1023

Lombardi, M., Alves, J., \& Lada, C. J. 2010, A\&A, 519, L7

Loren, R. B. 1989, ApJ, 338, 902

Mac Low, M.-M., \& Ossenkopf, V. 2000, A\&A, 353, 339

Mac Low, M.-M., \& Klessen, R. 2004, Rev. Mod. Phys., 76, 125

Matzner, C. D. 2002, ApJ, 566, 302

Men'shchikov, A., André, P., Didelon, P., et al. 2010, A\&A, 518, L106 (special issue Herschel)

Miville-Deschenes, M.-A., Joncas, G., Falgarone, E., \& Boulanger, F. 2003, A\&A, 411, 109

Molinari, S., Swinyard, B., Bally, J., et al. 2010, A\&A, 518, L100 (special issue Herschel)

Motte, F., André, P., \& Neri, R. 1998, A\&A, 336, 150

Motte, F., Bontemps, S., Schilke P., Schneider, N., \& Menten, K. 2007, A\&A, 476,1243

Motte, F., Zavagno A., Bontemps S., et al. 2010, A\&A, 518, L77 (special issue Herschel)

Nakamura, F., \& Li, Z.-Y. 2007, ApJ, 662, 395

Nakamura, F., \& Li, Z.-Y. 2008, ApJ, 687, 354

Offner, S. S. R., Klein, R. I., McKee, C. F., \& Krumholz, M. R. 2009, ApJ, 703, 131

Ossenkopf, V., Klessen, R., \& Heitsch, F. 2001, A\&A, 379, 1005

Ossenkopf, V. 2002, A\&A, 391, 295

Ossenkopf, V., \& Mac Low, M.-M., A\&A, 2002, 390, 307

Ossenkopf, V., Krips, M., \& Stutzki, J. 2008a, A\&A, 485, 917

Ossenkopf, V., Krips, M., \& Stutzki, J. 2008b, A\&A, 485, 719

Padoan, P., Jones, J. T., \& Nordlund, A. A. 1997, ApJ, 474, 730

Padoan, P., Juvela, M., Bally, J., \& Nordlund, A. A. 2000, ApJ, 529, 259

Padoan, P., Boldyrev, S., Langer, W., \& Nordlund, A. A. 2003, ApJ, 583, 308

Peters, T., Banerjee, R., \& Klessen, R. S. 2008, Physica Scripta, T132, 014026

Peters, T., Banerjee, R., Klessen, R. S., et al. 2010a, ApJ, 711, 1017

Peters, T., Mac Low, M.-M., Banerjee, R., et al. 2010b, ApJ, 719, 831

Peters, T., Banerjee, R., Klessen, R. S., et al. 2010c, ApJ, 711, 1017

Peters, T., Banerjee, R., Klessen, R. S., \& Mac Low, M.-M. 2011, ApJ, 729, 72

Piepenbrink, A., \& Wendker, H. J. 1988, A\&A, 191, 313

Pineda, J. E., Goodmann, A., Acre, H. G., et al. 2010, ApJ, 712, 116

Quillen, A. C., Thorndike, S. L., Cunningham, A., et al. 2005, ApJ, 632, 941

Reipurth, B., \& Schneider, N. 2008, Handbook of star-forming regions, ASP, 39

Robin, A. C., Reylé, C., Derrire, S., \& Picaud, S. 2003, A\&A, 409, 523

Scalo, J. M. 1987, Proceedings of the Symposium, Grand Teton National Park (Dordrecht: D. Reidel Publishing Co.), 349

Scalo, J., \& Elmegreen, B. G. 2004, ARA\&A, 42, 275

Schneider, N., \& Brooks, K. J. 2004, PASA, 21, 290

Schneider, N., Bontemps, S., Simon, R., et al. 2006, A\&A, 458, 855

Schneider, N., Simon, R., Bontemps, S., Comerón, F., \& Motte, F. 2007, A\&A, 474,873

Simon, R., Jackson, J. M., Clemens, D. M., Bania, T. M., \& Heyer, M. H. 2001, ApJ, 551, 747

Stutzki, J. 2001, Ap\&SS, 277, 39

Stutzki, J., \& Güsten, R. 1990, ApJ, 356, 513

Stutzki, J., Bensch, F., Heithausen, A., Ossenkopf, V., \& Zielinsky, M. 1998, A\&A, 336, 697

Sun, K., Kramer, C., Ossenkopt, V., Bensch, F., \& Stutzki, J. 2006, A\&A, 451, 539

Tachihara, K., Neuhäuser, R., \& Toyoda, S. 2001, Astr. Gesellschaft Abstract Series, 18, 72

Testi, L., \& Sargent, A. I. 1998, ApJ, 508, L91

Uyaniker, B., Fürst, E., Reich, W., Aschenbach, B., \& Wielebinski, R. 2001, A\&A, 371, 675

Vazquez-Semadeni, E., Ballesteros-Paredes, J., \& Rodriguez, L. F. 1997, ApJ, 474,292

Vázquez-Semadeni, E., Ballesteros-Paredes, J., \& Klessen, R. S. 2003, ApJ, 585, L131

Vazquez-Semadeni, Gomez, G. C., Jappsen, A. K., et al. 2007, ApJ, 657, 870

Vazquez-Semadeni, E., Gonzales, R. F., \& Ballesteros-Paredes, J., et al. 2008, MNRAS, 390, 769

Walder, R., \& Folini, D. 1998, A\&A, 330, L21

Wang, P., Li, Z.-Y., Abel, T., \& Nakamura, F. 2010, ApJ, 709, 27

Williams, J., Blitz, L., \& Stark, T. 1995, ApJ, 451, 252

Zuckerman, B., \& Palmer, P. 1974, ARA\&A, 12, 279 